Article

\title{
The Parotoid Gland Secretion from Peruvian Toad Rhinella horribilis (Wiegmann, 1833): Chemical Composition and Effect on the Proliferation and Migration of Lung Cancer Cells
}

\author{
Guillermo Schmeda-Hirschmann ${ }^{1, *} \mathbb{C}^{\mathbb{D}}$, Jean Paulo de Andrade ${ }^{1,2}$, Marilú Roxana Soto-Vasquez ${ }^{3}$, \\ Paul Alan Arkin Alvarado-García ${ }^{4}$, Charlotte Palominos 5,6, Sebastián Fuentes-Retamal 5,6 (D), \\ Mathias Mellado ${ }^{5,6}$, Pablo Correa ${ }^{5,6}$ and Félix A. Urra ${ }^{5,6, * \mathbb{D}}$ \\ 1 Laboratorio de Química de Productos Naturales, Instituto de Química de Recursos Naturales, \\ Universidad de Talca, Campus Lircay, Talca 3460000, Chile; jean.deandrade@utalca.cl \\ 2 Núcleo Científico Multidisciplinario, Dirección de Investigación, Universidad de Talca, Campus Lircay, \\ Talca 3460000, Chile \\ 3 Laboratorio de Farmacognosia, Facultad de Farmacia y Bioquímica, Universidad Nacional de Trujillo, \\ Trujillo 13011, Peru; msoto@unitru.edu.pe \\ 4 Facultad de Medicina, Universidad Privada Antenor Orrego, Trujillo 13001, Peru; palvaradog1@upao.edu.pe \\ 5 Laboratorio de Plasticidad Metabólica y Bioenergética, Programa de Farmacología Clínica y Molecular, \\ Instituto de Ciencias Biomédicas, Facultad de Medicina, Universidad de Chile, Santiago 8380453, Chile; \\ palominos.ch@gmail.com (C.P.); sebastianfuentesr@gmail.com (S.F.-R.); \\ mathignaciomellado@gmail.com (M.M.); pablo.correa.r@gmail.com (P.C.) \\ 6 Network for Snake Venom Research and Drug Discovery, Santiago 7800003, Chile \\ * Correspondence: schmeda@utalca.cl (G.S.-H.); felixurraf@u.uchile.cl (F.A.U.); \\ Tel.: +56-712-200-288 (G.S.-H.); +56-22-978-6066 (F.A.U.)
}

Received: 17 August 2020; Accepted: 9 September 2020; Published: 22 September 2020

check for updates

\begin{abstract}
Since Rhinella sp. toads produce bioactive substances, some species have been used in traditional medicine and magical practices by ancient cultures in Peru. During several decades, the Rhinella horribilis toad was confused with the invasive toad Rhinella marina, a species documented with extensive toxinological studies. In contrast, the chemical composition and biological effects of the parotoid gland secretions (PGS) remain still unknown for R. horribilis. In this work, we determine for the first time 55 compounds from the PGS of $R$. horribilis, which were identified using HPLC-MS/MS. The crude extract inhibited the proliferation of A549 cancer cells with $\mathrm{IC}_{50}$ values of $0.031 \pm 0.007$ and $0.015 \pm 0.001 \mu \mathrm{g} / \mathrm{mL}$ at 24 and $48 \mathrm{~h}$ of exposure, respectively. Moreover, it inhibited the clonogenic capacity, increased ROS levels, and prevented the etoposide-induced apoptosis, suggesting that the effect of $R$. horribilis poison secretion was by cell cycle blocking before of G2/M-phase checkpoint. Fraction $B$ was the most active and strongly inhibited cancer cell migration. Our results indicate that the PGS of $R$. horribilis are composed of alkaloids, bufadienolides, and argininyl diacids derivatives, inhibiting the proliferation and migration of A549 cells.
\end{abstract}

Keywords: Rhinella horribilis; toad toxins; Peru; cell migration; proliferation; etoposide; bufadienolides

Key Contribution: This is the first comprehensive analysis of the chemical composition of parotoid gland secretions of Rhinella horribilis, a species previously confused with R. marina. 


\section{Introduction}

Toads are widely distributed in the different biotas of Peru with a higher number of species in the Amazonian and eastern Andes [1,2] and are less frequent in the dry coastal ecosystems. Toads were known by the Native Americans both for their role in controlling insects as well as by their toxic defense substances. These toad toxins are secreted from parotoid glands that in contact with oral mucosa or eyes can produce intoxication or death [3-5]. Ceramic representations of Rhinella toad species by Peruvian pre-Inca Chavin and Moche cultures [6,7] and current local magic traditions in northern Peru that involved these toads (Figure 1A,B) suggest the presence of bioactive compounds. Currently, no studies are reporting the chemical composition and biological effects of the parotid glands secretions (PGS) of Rhinella sp. toads of the northern coast of Peru. The PGS of South American toads include alkaloids, argininyl diacids, bufadienolides, and argininyl diacid derivatives from bufadienolides. The biological effects from these secretions include cardiotonic, cytotoxic, and antiproliferative activities, sometimes with a remarkable and selective effect on several cancer cell lines [8-10].

Non-small cell lung cancer (NSCLC) is the leading cause of cancer-related deaths globally [11]. The NSCLC tumors have high molecular heterogeneity, exhibiting gene mutations in B-Raf proto-oncogene, serine/threonine kinase (BRAF), KRAS proto-oncogene, and activating mutations of epidermal growth factor receptor (EGFR) [12]. Although recent advances in the development of new therapeutic strategies are focused on the reduction of proliferation and tumor growth $[13,14]$; reduced efforts have been put into identifying anti-invasive and anti-migratory drugs [15]. Promising uses of microtubule-targeting agents (MTA) have been proposed for NSCLC treatment in advanced stages [13]. Beyond the known effects of MTA on cell cycle progression, the blocking the microtubules stabilization by these anti-cancer drugs produces disruption of cell protrusions and cytoskeleton, triggering inhibition of tumor-induced angiogenesis and cancer cell migration/invasion [16].

Previously, we and others have described that the PGS of Rhinella species produces inhibition of proliferation by cell cycle arrest and induction of apoptosis $[9,17,18]$, being the bufadienolides a promising source of anti-cancer chemical scaffolds [19]. The South American toad defense secretions consist of a complex mixture of argininyl diacids, bufadienolides argininyl diacids, bufadienolides, indolealkylamines, and peptides. The components less investigated so far are the peptides. Bioactive peptides also are considered a potential class of anticancer natural products, which inhibit cancer proliferation [20,21], angiogenesis [22], and induce selective apoptosis [23].

Little is known on the PGS composition and bioactivity of toad defensive compounds for the western South American Andes. No information is available on the secretion constituents of the recently known species of the northern Peru Rhinella horribilis (Figure 1C), which was extensively confused with Rhinella marina [24], a highly invasive species with well-known toxicological effects and bioactivities $[8,18,25,26]$.

In this work, we report for the first time the chemical composition of the PGS from the Peruvian toad $R$. horribilis and describe the effect of the whole secretion and their main fractions on human lung cancer cells. 

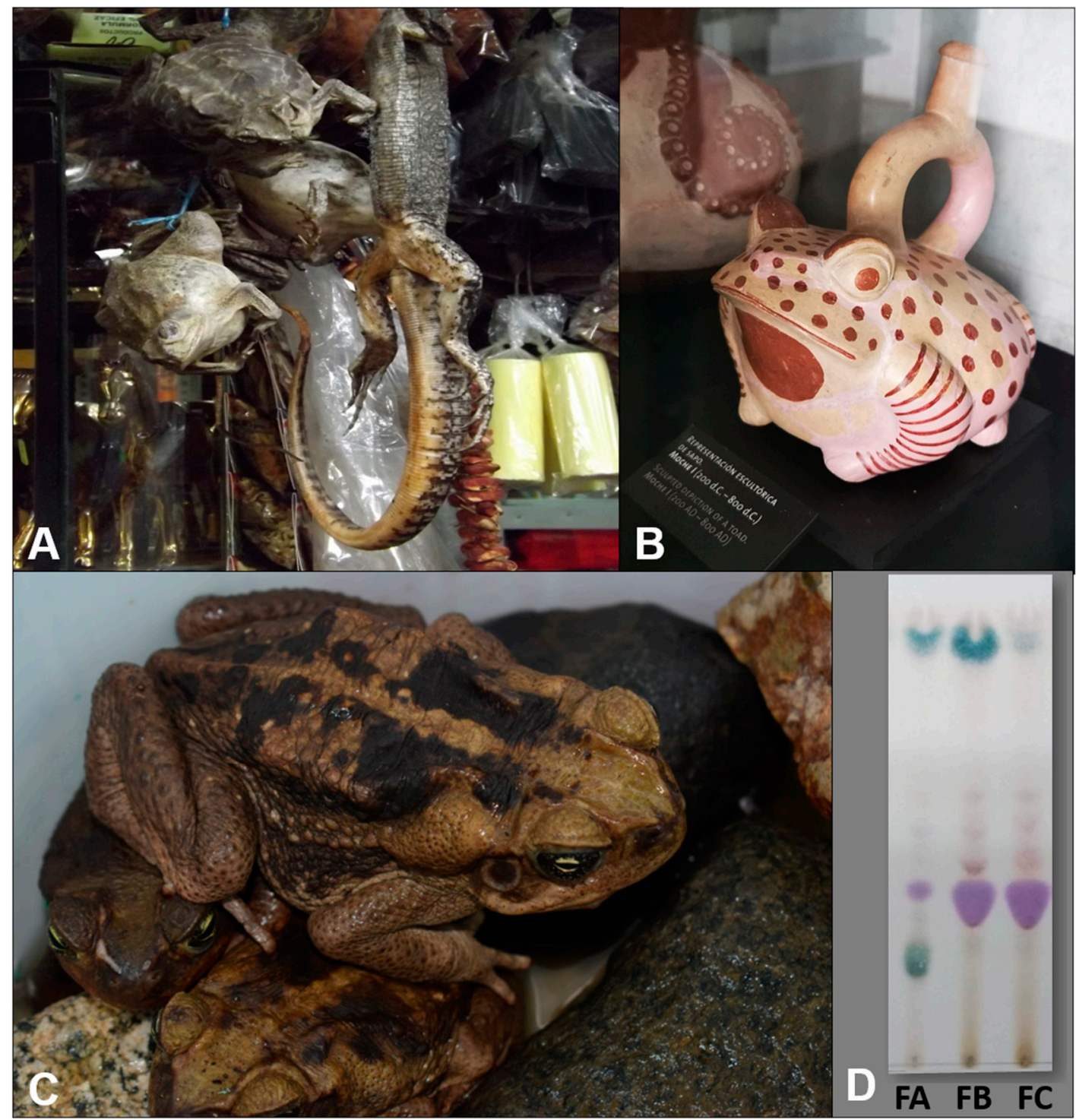

Figure 1. Representations of Rhinella species in traditional medicine and magical practices in Peru: (A) frogs and lizards on sale at the "Mercado de Brujos" (Witch's market), Chichlayo, Peru; (B) Moche ceramic representing a frog; (C) adult specimen of Rhinella horribilis (Wiegmann, 1833), Peru; (D) thin layer chromatographic analysis (TLC) of the Sephadex LH-20 permeation showing the fraction pools A (FA; 27-30), B (FB; 31-38), and C (FC; 39-57) of PGS from R. horribilis. Adsorbent: silica gel; solvent system: EtOAc:isopropanol: $\mathrm{NH}_{4} \mathrm{OH}$ (9:7:4). The plate was sprayed with a freshly prepared $p$-anisaldehyde solution reagent followed by heating up to $105^{\circ} \mathrm{C}$ for maximum visualization of spots.

\section{Results and Discussion}

\subsection{Fractionation of Parotoid Gland Secretions of R. horribilis}

The crude secretions were fractionated into a group of constituents according to molecular size by permeation on Sephadex LH-20. The sample $(660 \mathrm{mg})$ was loaded into a Sephadex LH-20 column (column length: $28.5 \mathrm{~cm}$; internal diameter: $3.5 \mathrm{~cm}$; filled with Sephadex ${ }^{\circledR} \mathrm{LH}-20$, Merck KGaA, Darmstadt, Germany). The eluent was methanol:chloroform (1:1). The void volume was $300 \mathrm{~mL}$ and 95 fractions of $3 \mathrm{~mL}$ each were collected. After TLC analysis (solvent system: ethanol:isopropanol:ammonium hydroxide 9:7:4 v/v/v), fractions with similar patterns were pooled as follows: fraction 11-26 (182.6 mg), fraction 27-30 (96.6 mg), fraction 31-38 (116.8 mg), fraction 39-57 (77.3 mg), fraction 58-69 (12 mg), and fraction 70-95 (12 mg) (Figure 1D). The pooled fractions 
containing most of the constituents were further analyzed by ${ }^{1} \mathrm{H}$ NMR to obtain structural information on the group of compounds and for further examination by HPLC-MS/MS.

\section{2. ${ }^{1} H$ NMR Analysis}

The ${ }^{1} \mathrm{HNMR}$ spectrum of the fractions 27-30 (Pool A) showed the characteristic signals for the $\mathrm{H}-21, \mathrm{H}-22$, and $\mathrm{H}-23$ protons of the bufadienolide $\alpha$-pyrone ring at $\delta 7.37-7.48,7.86-8.00$, and $6.25-6.32$ ppm, respectively. Minor compounds of the pool presented a singlet at $\delta 9.67,9.58$, and $9.48 \mathrm{ppm}$, corresponding to an aldehyde function. The less abundant bufadienolides in the complex mixture displayed a singlet or broad singlet at $\delta 10.10,10.08,10.07$, and $10.03 \mathrm{ppm}$, compatible with a second group of oxidized derivatives with an aldehyde group. According to Kamano et al. [27], the aldehyde signal resonates at $\delta 10.06 \mathrm{ppm}$ for hellebrigenin and $\delta 9.49 \mathrm{ppm}$ for resibufagin. The ${ }^{1} \mathrm{HNMR}$ spectrum of our sample supports the presence of a mixture of compounds belonging mainly to the bufalin and marinobufagin series, with the aldehydes and further oxidized bufadienolides as minor constituents. The main bufadienolides in the fraction are marinobufagin and bufalin argininyl diacids. The HPLC-MS/MS analysis (Section 2.3) confirmed the occurrence of several hellebrigenin and resibufagin argininyl diacids, supporting the NMR assignments. The Pool B, including fractions 31-38, shows a mixture of argininyl diacids, free bufadienolides, and minor amounts of bufadienolides argininyl diacids. The main groups of the components were resibufagin, telocinobufagin, marinobufagin, bufalin, and resibufogenin derivatives. The identity was established by HPLC-MS/MS analysis (see Section 2.3). The ${ }^{1} \mathrm{HNMR}$ analysis of the fraction 39-57 (Pool C) showed a main compound with $\mathrm{H}$ signals at $\delta 7.32 \mathrm{~d}(8.4)(1 \mathrm{H}), 7.12 \mathrm{~s}(1 \mathrm{H}), 6.86 \mathrm{~d}(8.4)(1 \mathrm{H}), 4.02 \mathrm{br} \mathrm{t}(5.6)$ $(2 \mathrm{H}), 3.71 \mathrm{~s}(6 \mathrm{H})$ and $3.31 \mathrm{ppm}(\mathrm{br} \mathrm{t}, 5.6)$, in agreement with dehydrobufotenine (DHB) (compound A2). Additional signals support the presence of a mixture of argininyl diacids and minor amounts of bufadienolides. HPLC-MS/MS analysis of the fraction confirmed the occurrence of DHB as the main constituent and the presence of adipyl-, pimeloyl- and suberoylarginine. The ${ }^{1} \mathrm{HNMR}$ analysis of the pool of fractions 58-69 and 70-95 showed the characteristic signals for a mixture of argininyl diacids and was not further investigated.

\subsection{HPLC-DAD-Q-TOF-MS/MS Analysis}

The crude PGS and the main fraction pools A, B, and C were analyzed by high performance liquid chromatography (HPLC) coupled to a diode-array detector (DAD) and to a quadrupole time-of-flight mass spectrometry/mass spectrometry detector (Q-TOF-MS/MS) to identify the constituents of the complex mixtures (Table 1). Detected and calculated $[\mathrm{M}+\mathrm{H}]^{+}$ions were compared and the error was calculated for the tentative identification of the different compounds. Data analyses followed previous work on toad poison secretions $[9,10,18,28-31]$.

\subsubsection{Compounds Identified: Argininyl Diacids}

In the HPLC-MS/MS analysis (Table 1 and Figure 2), six compounds eluting in the first third of the chromatographic run showed the loss of a diacid from the positively charged ion and arginine at $\mathrm{m} / \mathrm{z} 175$ as the common amino acid in the structural series. The difference in the compounds 1-6 (Figure 3) was the number of $\mathrm{CH}_{2}$ units in the diacid moiety. The compounds were identified as adipoyl-, pimeloyl-, suberoyl-, azelayl-, sebacyl- and undecadienoyl arginine, respectively. Argininyl diacids were described in the poison secretions of other South American Rhinella species, including R. marina [18], R. schneideri [9,10], R. ornata and R. scitula [9]. For all argininyl diacids and N-diacid argininyl bufadienolides, extracted ion chromatograms at $m / z 303,317,331,345,359$ and 373 allowed to confirm the occurrence of adipyl-, pimeloyl-, suberoyl-, azeloyl-, sebacyl- and undecadienoyl arginine esters, respectively. 

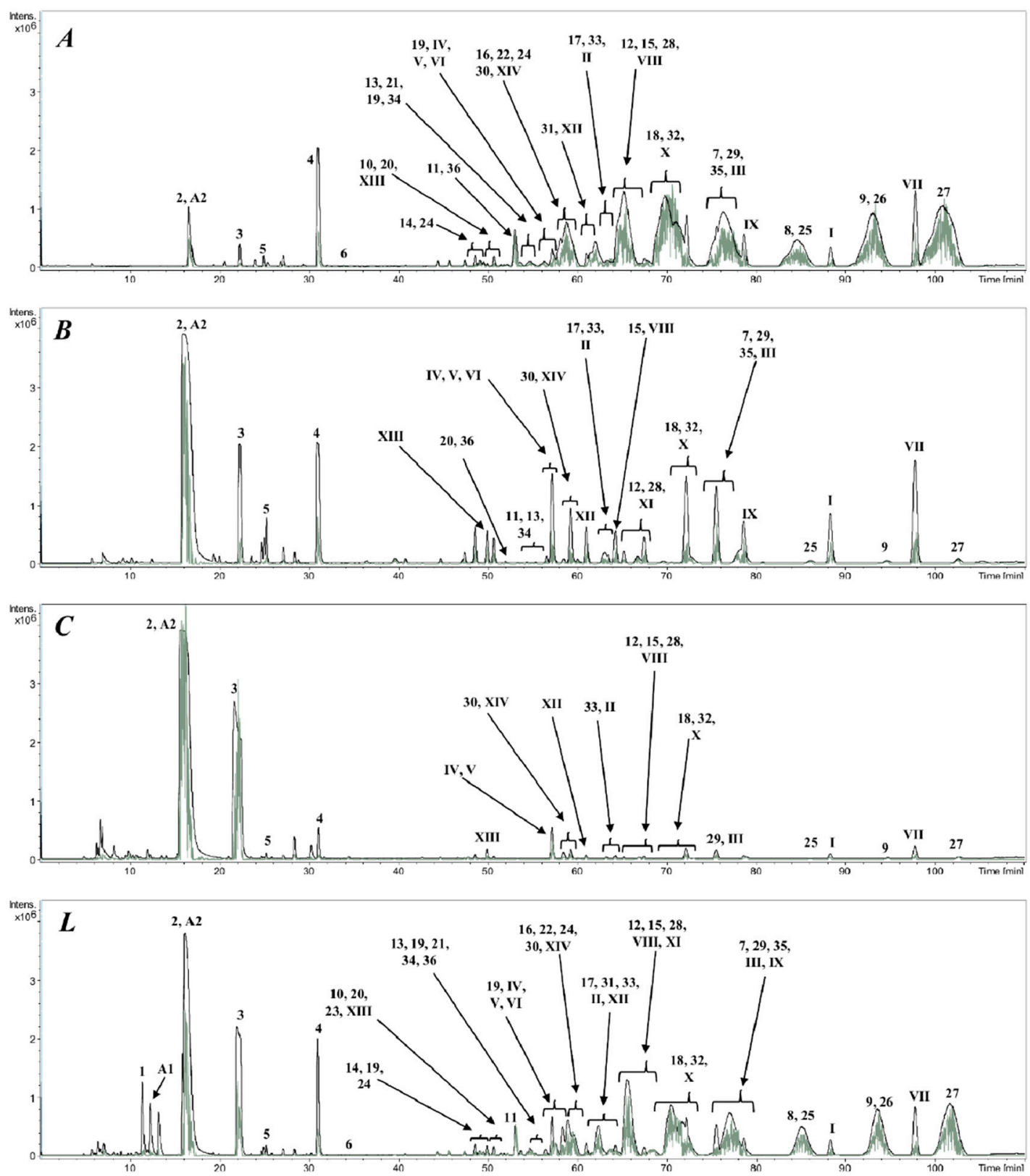

Figure 2. HPLC-MS/MS chromatogram of the fractions 27-30 (A), 31-38 (B), 39-57 (C), and the lyophilized crude extract (L) of the parotoid gland secretions from Peruvian Rhinella horribilis. Total ion current (TIC) (black line) and $\mathrm{MS}^{2}$ (gray line). Detection in the positive ion mode. 
Table 1. HPLC-MS/MS data (positive ion mode) of the defense compounds of the lyophilized crude extract of parotoid gland secretion (L) from the Peruvian Rhinella horribilis and the fractions 27-30 (A), 31-38 (B), and 39-57 (C). Abbreviations: $\mathrm{N}^{\circ}=$ compound number, for the structures see Figures 3-5; Rt $=$ Retention time; Sample: fractions 27-30 (A), 31-38 (B), 39-57 (C), lyophilized crude extract (L).

\begin{tabular}{|c|c|c|c|c|c|c|c|}
\hline $\mathbf{N}^{\circ}$ & Rt (min) & Sample & $\begin{array}{l}{[\mathrm{M}+\mathrm{H}]^{+}} \\
\text {Detected }\end{array}$ & $\begin{array}{l}{[\mathrm{M}+\mathrm{H}]^{+}} \\
\text {Expected }\end{array}$ & $\begin{array}{l}\text { Error } \\
(\mathrm{ppm})\end{array}$ & MS/MS Fragmentation & Tentative Identification \\
\hline 1 & $11.4-11.9$ & $\mathrm{~L}$ & 303.1624 & 303.1663 & 12.8 & $\begin{array}{c}303.1624(<1), 244.1169(32), 222.1210(14), 175.1184(27), 158.0852(22), \\
130.1935(14), 128.0688(26), 116.0685(100), 95.0600(39) .\end{array}$ & $\begin{array}{l}\text { Adipoyl arginine } \\
\mathrm{C}_{12} \mathrm{H}_{23} \mathrm{~N}_{4} \mathrm{O}_{5}^{+}[28]\end{array}$ \\
\hline A1 & 12.8 & $\mathrm{~L}$ & 176.0685 & 176.0666 & 10.8 & $\begin{array}{c}176.0688(<1), 159.0660(17), 143.0720(9), 133.0536(16), 117.0560(52) \\
115.0533(100), 115.0533(100), 105.0673(31), 89.0368(17)\end{array}$ & $\begin{array}{l}\text { Guanidinosuccinic acid } \\
\qquad \mathrm{C}_{5} \mathrm{H}_{10} \mathrm{~N}_{3} \mathrm{O}_{4}^{+}[28]\end{array}$ \\
\hline 2 & $15.6-17.0$ & $\mathrm{~A}, \mathrm{~B}, \mathrm{C}, \mathrm{L}$ & 317.1804 & 317.1819 & 4.7 & $\begin{array}{c}317.1804(<1), 273.4732(40), 194.1169(52), 158.0895 \text { (65), } 125.0623(87), \\
112.0849(100)\end{array}$ & Pimeloyl arginine $\mathrm{C}_{13} \mathrm{H}_{25} \mathrm{~N}_{4} \mathrm{O}_{5}{ }^{+}[28]$ \\
\hline A2 & $15.7-17.2$ & A, B, C, L & 203.1178 & 203.1179 & 0.5 & $\begin{aligned} 203.1178(<1), 188.0932(50), & 173.0708(100), 145.0764(9), 118.0667 \text { (10), } \\
& 89.0364(16)\end{aligned}$ & Dehydrobufotenine $\mathrm{C}_{12} \mathrm{H}_{15} \mathrm{~N}_{2} \mathrm{O}^{+}[28]$ \\
\hline 3 & $21.8-22.7$ & $\mathrm{~A}, \mathrm{~B}, \mathrm{C}, \mathrm{L}$ & 331.1962 & 331.1976 & 4.2 & $331.1980(20), 272.1486(100), 172.1057(86), 125.0603(48), 112.0879$ (46) & $\begin{array}{l}\text { Suberoyl arginine } \\
\mathrm{C}_{14} \mathrm{H}_{27} \mathrm{~N}_{4} \mathrm{O}_{5}^{+}[28]\end{array}$ \\
\hline 5 & $25.2-25.5$ & $\mathrm{~A}, \mathrm{~B}, \mathrm{C}, \mathrm{L}$ & 359.2260 & 359.2289 & 8.0 & $\begin{array}{c}359.2366 \text { (21), } 272.1458 \text { (100), } 246.4343 \text { (17), } 226.1422 \text { (20), } 203.1506 \text { (33), } \\
116.0996 \text { (35) }\end{array}$ & $\begin{array}{l}\text { Sebacyl arginine isomer } \\
\quad \mathrm{C}_{16} \mathrm{H}_{31} \mathrm{~N}_{4} \mathrm{O}_{5}^{+}[28]\end{array}$ \\
\hline 4 & $30.9-31.5$ & $\mathrm{~A}, \mathrm{~B}, \mathrm{C}, \mathrm{L}$ & 345.2123 & 345.2132 & 2.6 & $\begin{array}{c}345.2100(19), 286.1628(27), 250.1529(85), 175.1197(58), 158.0932(44), \\
139.0761(42), 116.0709(100)\end{array}$ & Azelayl arginine $\mathrm{C}_{15} \mathrm{H}_{29} \mathrm{~N}_{4} \mathrm{O}_{5}{ }^{+}[28]$ \\
\hline 6 & $34.0-34.1$ & A, L & 373.2417 & 373.2445 & 8.3 & $\begin{array}{c}373.2341 \text { (34), } 286.1592 \text { (100), } 254.1458(30), 203.1485(40), 172.1096(40), \\
116.0702(75)\end{array}$ & $\begin{array}{l}\text { Undecadienoyl arginine } \\
\qquad \mathrm{C}_{17} \mathrm{H}_{33} \mathrm{~N}_{4} \mathrm{O}_{5}^{+}[28]\end{array}$ \\
\hline 24 & $48.8-49.3$ & A, L & 745.4000 & 745.4018 & 2.4 & $\begin{array}{c}745.3983(78), 727.3875(13), 434.7749(6), 331.1942(100), 313.1949(11), \\
278.1473(33), 250.1558(25), 195.1161(17), 158.0890(17), 117.0713(31), \\
112.0840(58), 91.0538(27)\end{array}$ & $\begin{array}{l}\text { 3-(N-suberoyl argininyl) } \\
\text { Hydroxyhellebrigenin isomer } 1 \\
\quad \mathrm{C}_{38} \mathrm{H}_{57} \mathrm{~N}_{4} \mathrm{O}_{11}{ }^{+}[28,29]\end{array}$ \\
\hline 19 & $49.1-49.2$ & $\mathrm{~L}$ & 731.4225 & 731.4226 & 0.1 & 331.1932 (100), $314.1670(18), 278.1441(25), 175.1128(10)$ & $\begin{array}{l}\text { 3-(N-suberoyl argininyl) Hellebrigenol } \\
\quad \text { isomer } 1 \mathrm{C}_{38} \mathrm{H}_{59} \mathrm{~N}_{4} \mathrm{O}_{10}{ }^{+}[28]\end{array}$ \\
\hline 14 & $49.3-49.7$ & A, L & 715.3888 & 715.3913 & 3.5 & $\begin{array}{c}715.3906(40), 697.3787 \text { (5), } 363.1930(5), 345.1796(2), 317.1788 \text { (100), } 264.1340 \\
\text { (7), } 175.1181(4), 112.0878 \text { (11) }\end{array}$ & $\begin{array}{l}\text { 3-(N-pimeloyl argininyl) } \\
\text { Desacetylcinobufotalin } \mathrm{C}_{37} \mathrm{H}_{55} \mathrm{~N}_{4} \mathrm{O}_{10}+[32]\end{array}$ \\
\hline 10 & $49.8-50.1$ & A, L & 687.3952 & 687.3964 & 1.7 & $\begin{array}{c}687.3984(46), 669.3618(4), 385.2361(5), 303.1627(100), 268.1292(11) \\
250.1171(13), 105.0685(12)\end{array}$ & $\begin{array}{l}\text { 3-(N-adipoyl argininyl) Gamabufotalin } \\
\qquad \mathrm{C}_{36} \mathrm{H}_{55} \mathrm{~N}_{4} \mathrm{O}_{9}^{+}[28,32]\end{array}$ \\
\hline XIII & $50.6-50.9$ & $\mathrm{~A}, \mathrm{~B}, \mathrm{C}, \mathrm{L}$ & 417.2262 & 417.2272 & 2.4 & $\begin{array}{c}417.2286(100), 399.2198(18), 381.2053(50), 363.1946(34), 345.1840(33), \\
209.0929(62), 165.0687(62), 103.0526(89), 91.0544(94)\end{array}$ & Arenobufagin $\mathrm{C}_{24} \mathrm{H}_{33} \mathrm{O}_{6}^{+}[31]$ \\
\hline 23 & $50.8-50.9$ & $\mathrm{~L}$ & 731.3845 & 731.3862 & 2.3 & $\begin{array}{l}731.3845(35), 439.2103(11), 317.1809(100), 300.1562(16), 264.1334(20) \\
178.0778(9), 155.0880(13)\end{array}$ & $\begin{array}{l}\text { 3-(N-pimeloyl argininyl) } \\
\text { Hydroxyhellebrigenin } \mathrm{C}_{37} \mathrm{H}_{55} \mathrm{~N}_{4} \mathrm{O}_{11}{ }^{+} \text {[28] }\end{array}$ \\
\hline 20 & $51.5-51.7$ & $\mathrm{~A}, \mathrm{~B}, \mathrm{~L}$ & 701.3741 & 701.3756 & 2.1 & $\begin{array}{c}701.3747 \text { (96), } 683.3639(11), 665.3641(2), 381.2004(3), 364.1916(5), 363.1984 \\
(4), 335.1984(18), 317.1910(3), 303.1640(100), 268.1239(14), 250.1194(17) \\
175.1192(3), 129.0689(16), 91.0528(31)\end{array}$ & $\begin{array}{l}\text { 3-(N-adipoyl argininyl) Hellebrigenin } \\
\qquad \mathrm{C}_{36} \mathrm{H}_{53} \mathrm{~N}_{4} \mathrm{O}_{10}{ }^{+}[32]\end{array}$ \\
\hline 36 & $52.1-52.4$ & $\mathrm{~A}, \mathrm{~B}, \mathrm{~L}$ & 743.3854 & 743.3862 & 2.3 & $\begin{array}{l}743.3860(100), 725.3687(7), 377.1703(9), 331.1968(99), 278.1481(26), 250.1546 \\
\quad(23), 175.1230(7), 165.0706(14), 128.0629(22), 112.0881(24), 910533(25)\end{array}$ & $\begin{array}{l}\text { 3-(N-suberoyl argininyl) } \\
\text { Hydroxybufotalinin isomer } 1 \\
\quad \mathrm{C}_{38} \mathrm{H}_{55} \mathrm{~N}_{4} \mathrm{O}_{11}{ }^{+}[28]\end{array}$ \\
\hline
\end{tabular}


Table 1. Cont.

\begin{tabular}{|c|c|c|c|c|c|c|c|}
\hline $\mathbf{N}^{\circ}$ & Rt (min) & Sample & $\begin{array}{l}{[\mathrm{M}+\mathrm{H}]^{+}} \\
\text {Detected }\end{array}$ & $\begin{array}{c}{[\mathrm{M}+\mathrm{H}]^{+}} \\
\text {Expected }\end{array}$ & $\begin{array}{c}\text { Error } \\
(\mathrm{ppm})\end{array}$ & MS/MS Fragmentation & Tentative Identification \\
\hline 11 & $53.3-54.1$ & $\mathrm{~A}, \mathrm{~B}, \mathrm{~L}$ & 701.4062 & 701.4120 & 8.2 & $\begin{array}{c}701.4080 \text { (95), } 683.3979(2), 385.2287(4), 317.1767 \text { (100), } 264.1285(33) \\
236.1363(8), 105.0669(20)\end{array}$ & $\begin{array}{l}\text { 3-(N-pimeloyl argininyl) Gamabufotalin } \\
\qquad \mathrm{C}_{37} \mathrm{H}_{57} \mathrm{~N}_{4} \mathrm{O}_{9}{ }^{+}[28]\end{array}$ \\
\hline 21 & $54.1-55.0$ & A, L & 715.3879 & 715.3913 & 4.8 & $\begin{array}{c}715.3864(100), 697.3839(7), 669.3725(3), 381.2027(5), 364.1992(1), 363.1928 \\
(5), 651.3676(6), 353.2058(2), 345.1816(3), 335.2013(8), 317.1787 \text { (71), } \\
282.1417(8), 264.1309(14), 236.1364 \text { (9), } 175.1165(8), 112.0861(23), 105.0702 \\
(17), 91.0535(27)\end{array}$ & $\begin{array}{l}\text { 3-(N-pimeloyl argininyl) Hellebrigenin } \\
\qquad \mathrm{C}_{37} \mathrm{H}_{55} \mathrm{~N}_{4} \mathrm{O}_{10}{ }^{+}[32]\end{array}$ \\
\hline 19 & $54.2-54.6$ & A, L & 731.4171 & 731.4226 & 7.5 & $\begin{array}{c}731.4143 \text { (72), } 363.1946 \text { (12), } 331.1891(100), 278.1446 \text { (35), } 250.1450(15), \\
175.1177(17), 112.0873(23)\end{array}$ & $\begin{array}{l}\text { 3-(N-suberoyl argininyl) Hellebrigenol } \\
\text { isomer } 2 \mathrm{C}_{38} \mathrm{H}_{59} \mathrm{~N}_{4} \mathrm{O}_{10}{ }^{+}\end{array}$ \\
\hline 13 & $54.3-54.8$ & $\mathrm{~A}, \mathrm{~B}, \mathrm{~L}$ & 701.3728 & 701.3756 & 4.0 & $\begin{array}{l}701.3721(100), 684.3766(1), 610.9012(1), 399.2135(6), 381.2023(2), 353.2069 \\
\quad(2), 335.1959(1), 303.1635(89), 286.1387(9), 268.1256(15), 250.1156(17) \\
175.1180(11), 116.0692(16), 112.0878(15), 105.0691(13), 91.0537(10)\end{array}$ & $\begin{array}{l}\text { 3-(N-adipoyl argininyl) Arenobufagin } \\
\qquad \mathrm{C}_{36} \mathrm{H}_{53} \mathrm{~N}_{4} \mathrm{O}_{10}{ }^{+}[32]\end{array}$ \\
\hline 36 & $54.7-54.9$ & A, L & 743.3825 & 743.3862 & 4.9 & $\begin{array}{c}743.3829(65), 349.1888(8), 331.1935(100), 296.1560(9), 278.1480(25) \\
250.1528(55), 233.0955(4), 179.0855(11), 112.0869(34), 91.0555(22)\end{array}$ & $\begin{array}{l}\text { 3-(N-suberoyl argininyl) } \\
\text { Hydroxybufotalinin isomer } 2 \\
\mathrm{C}_{38} \mathrm{H}_{55} \mathrm{~N}_{4} \mathrm{O}_{11}{ }^{+}[28]\end{array}$ \\
\hline 34 & $55.1-55.4$ & $\mathrm{~A}, \mathrm{~B}, \mathrm{~L}$ & 713.3706 & 713.3756 & 7.0 & $\begin{array}{c}713.3715(100), 695.3598(3), 667.3739(4), 317.1781(88), 300.1509(22) \\
282.1434(12), 264.1334(12), 236.1370(16), 209.0860(5), 179.0818(10), 175.1225 \\
\text { (7), } 112.0855(35), 91.0524(43)\end{array}$ & $\begin{array}{l}\text { 3-(N-pimeloyl argininyl) } \\
\text { Bufotalinin } \mathrm{C}_{37} \mathrm{H}_{53} \mathrm{~N}_{4} \mathrm{O}_{10}{ }^{+}[28]\end{array}$ \\
\hline VI & $56.5-56.7$ & $\mathrm{~A}, \mathrm{~B}, \mathrm{~L}$ & 419.2400 & 419.2428 & 6.6 & $\begin{array}{c}419.2400(5), 401.2318(30), 365.2088(63), 347.2036(36), 319.1615(33), \\
213.1616(27), 91.0531(51)\end{array}$ & Hellebrigenol $\mathrm{C}_{24} \mathrm{H}_{35} \mathrm{O}_{6}{ }^{+}$[32] \\
\hline $\begin{array}{l}\text { IV } \\
\text { or } \\
\text { V }\end{array}$ & $57.0-57.6$ & $\mathrm{~A}, \mathrm{~B}, \mathrm{C}, \mathrm{L}$ & 417.2244 & 417.2272 & 6.7 & $\begin{array}{c}417.2226(100), 399.2122(35), 371.2176(4), 353.2056(3), 335.1972 \text { (5), } 255.0976 \\
(2), 175.0722(4)\end{array}$ & $\begin{array}{l}\text { Bufarenogin or } \psi \text {-Bufarenogin } \\
\qquad \mathrm{C}_{24} \mathrm{H}_{33} \mathrm{O}_{6}{ }^{+} \text {[32] }\end{array}$ \\
\hline 19 & $57.0-57.8$ & A, L & 731.4184 & 731.4226 & 5.7 & $\begin{array}{l}731.4189(55), 713.4048(5), 683.3909(10), 665.3843(4), 331.1919(100), \\
278.1468(10), 250.1475(9), 175.1179(4), 112.0854(20)\end{array}$ & $\begin{array}{l}\text { 3-(N-suberoyl argininyl) Hellebrigenol } \\
\text { isomer } 3 \\
\mathrm{C}_{38} \mathrm{H}_{59} \mathrm{~N}_{4} \mathrm{O}_{10}{ }^{+}[28]\end{array}$ \\
\hline 16 & $57.9-58.2$ & A, L & 687.3947 & 687.3964 & 2.4 & $\begin{array}{c}687.3960(27), 669.3813(7), 303.1641(100), 250.1177(13), 175.1446(8), \\
145.0968(14), 91.0533(17)\end{array}$ & $\begin{array}{l}\text { 3-(N-adipoyl argininyl) Telocinobufagin } \\
\qquad \mathrm{C}_{36} \mathrm{H}_{55} \mathrm{~N}_{4} \mathrm{O}_{9}{ }^{+}[28]\end{array}$ \\
\hline 24 & $58.1-58.4$ & A, L & 745.4001 & 745.4018 & 2.3 & $\begin{array}{c}745.4017(11), 683.4230(11), 345.2155(10), 331.1923(100), 303.1952(8), \\
278.1492(10), 175.1148(15), 112.0836(35)\end{array}$ & $\begin{array}{l}\text { 3-(N-suberoyl argininyl) } \\
\text { Hydroxyhellebrigenin isomer } 2 \\
\quad \mathrm{C}_{38} \mathrm{H}_{57} \mathrm{~N}_{4} \mathrm{O}_{11}{ }^{+}[28]\end{array}$ \\
\hline 30 & $58.3-59.0$ & A, B, C, L & 685.3780 & 685.3807 & 3.9 & $\begin{array}{c}685.3757(28), 669.3790(5), 667.3653(3), 365.2086(3), 349.2058(4), 303.1615 \\
(100), 268.1240(12), 250.1140(12), 175.1140(6), 105.0678(13)\end{array}$ & $\begin{array}{l}\text { 3-(N-adipoyl argininyl) Marinobufagin } \\
\qquad \mathrm{C}_{36} \mathrm{H}_{53} \mathrm{~N}_{4} \mathrm{O}_{9}+[28]\end{array}$ \\
\hline 22 & $58.4-60.0$ & A, L & 729.4021 & 729.4069 & 6.5 & $\begin{array}{l}729.4010(100), 711.3899(8), 693.3760(2), 683.3970(2), 363.1912(4), 331.1926 \\
(68), 278.1459(16), 250.1516(16), 175.1188(8), 112.0861(27), 91.0534(27)\end{array}$ & $\begin{array}{l}\text { 3-(N-suberoyl argininyl) Hellebrigenin } \\
\mathrm{C}_{38} \mathrm{H}_{57} \mathrm{~N}_{4} \mathrm{O}_{10}{ }^{+}[28,29]\end{array}$ \\
\hline XIV & $59.0-59.6$ & $\mathrm{~A}, \mathrm{~B}, \mathrm{C}, \mathrm{L}$ & 417.2242 & 417.2272 & 7.2 & $\begin{array}{l}417.2244(62), 399.2122(26), 363.1903(30), 353.2077(26), 335.1959(73), \\
317.1874(10), 275.1754(16), 211.1433(33), 128.0608(71), 91.0527(100)\end{array}$ & Hellebrigenin $\mathrm{C}_{24} \mathrm{H}_{33} \mathrm{O}_{6}^{+}[28]$ \\
\hline 31 & $60.5-64.3$ & A, L & 699.3980 & 699.3964 & 2.3 & $\begin{array}{c}699.3971(38), 681.3823(7), 365.2102(4), 347.2001(4), 317.1806(100), 264.1314 \\
(11), 236.1361(6), 175.1198(5), 112.0883(10), 91.0523(10)\end{array}$ & $\begin{array}{l}\text { 3-(N-pimeloyl argininyl) Marinobufagin } \\
\left.\qquad \mathrm{C}_{37} \mathrm{H}_{55} \mathrm{~N}_{4} \mathrm{O}_{9}^{+}{ }^{+} 28\right]\end{array}$ \\
\hline
\end{tabular}


Table 1. Cont.

\begin{tabular}{|c|c|c|c|c|c|c|c|}
\hline $\mathbf{N}^{\circ}$ & Rt (min) & Sample & $\begin{array}{l}{[\mathrm{M}+\mathrm{H}]^{+}} \\
\text {Detected }\end{array}$ & $\begin{array}{l}{[\mathrm{M}+\mathrm{H}]^{+}} \\
\text {Expected }\end{array}$ & $\begin{array}{l}\text { Error } \\
(\mathrm{ppm})\end{array}$ & MS/MS Fragmentation & Tentative Identification \\
\hline XII & $60.8-61.4$ & $\mathrm{~A}, \mathrm{~B}, \mathrm{C}, \mathrm{L}$ & 415.2092 & 415.2115 & 5.5 & $\begin{array}{l}415.2085(22), 379.1865(8), 361.1792(12), 351.1927(30), 333.1820(26), \\
237.1619(28), 165.0669(30), 128.0609(64), 115.0530(79), 91.0534(100)\end{array}$ & Bufotalinin $\mathrm{C}_{24} \mathrm{H}_{31} \mathrm{O}_{6}{ }^{+}$[32] \\
\hline 17 & $62.4-64.7$ & A, B, L & 701.4097 & 701.4120 & 3.3 & $\begin{array}{c}701.4087(24), 683.3955(5), 349.2203(3), 317.1786(100), 264.1308(5), 236.1406 \\
\text { (3), } 175.1172(3), 105.0711(8)\end{array}$ & $\begin{array}{l}\text { 3-(N-pimeloyl argininyl) Telocinobufagin } \\
\qquad \mathrm{C}_{37} \mathrm{H}_{57} \mathrm{~N}_{4} \mathrm{O}_{9}^{+}[28]\end{array}$ \\
\hline 33 & $62.7-63.7$ & $\mathrm{~A}, \mathrm{~B}, \mathrm{C}, \mathrm{L}$ & 683.3682 & 683.3651 & 3.6 & $\begin{array}{c}683.3613(100), 665.3521(2), 335.1963(4), 303.1635(40), 286.1371(9), 268.1273 \\
\text { (8), } 250.1161(17), 226.1057(3), 175.1187(5), 112.0873(13)\end{array}$ & $\begin{array}{l}\text { 3-(N-adipoyl argininyl) } \\
\text { Marinobufagin-9,11-ene } \mathrm{C}_{36} \mathrm{H}_{51} \mathrm{~N}_{4} \mathrm{O}_{9}{ }^{+}\end{array}$ \\
\hline II & $62.9-63.4$ & $\mathrm{~A}, \mathrm{~B}, \mathrm{C}, \mathrm{L}$ & 403.2453 & 403.2479 & 6.4 & $\begin{array}{l}403.2445(30), 385.2335(58), 349.2120(44), 337.2111(41), 275.1742(25), \\
253.1888(39), 241.1922(49), 161.0968(29), 105.0785(46), 91.0546(100) \\
401.2295(34), 383.2866(3), 365.2081(15), 347.1970(20), 337.2165(14)\end{array}$ & Gamabufotalin $\mathrm{C}_{24} \mathrm{H}_{35} \mathrm{O}_{5}{ }^{+}[28,32]$ \\
\hline VIII & $64.0-64.7$ & $\mathrm{~A}, \mathrm{~B}, \mathrm{C}, \mathrm{L}$ & 401.2312 & 401.2323 & 2.7 & $\begin{array}{c}319.2024(10), 257.1163(21), 239.1059(32), 211.1096(27), 183.1154(24), \\
128.0626(86), 105.0700(85), 91.0547(100)\end{array}$ & Resibufaginol $\mathrm{C}_{24} \mathrm{H}_{33} \mathrm{O}_{5}{ }^{+}[31]$ \\
\hline 15 & $64.4-66.5$ & $\mathrm{~A}, \mathrm{~B}, \mathrm{C}, \mathrm{L}$ & 729.4052 & 729.4069 & 2.3 & $\begin{array}{c}729.4077 \text { (100), } 399.2156(5), 381.2065(2), 363.1959(2), 353.2100(1), 345.1862 \\
(1), 335.1983(2), 331.1959(83), 278.1487(25), 250.1541 \text { (18), } 175.1200(11) \\
112.0875(27)\end{array}$ & $\begin{array}{l}\text { 3-(N-suberoyl argininyl) } \\
\text { Desacetylcinobufotalin } \mathrm{C}_{38} \mathrm{H}_{57} \mathrm{~N}_{4} \mathrm{O}_{10}+[29]\end{array}$ \\
\hline 12 & $66.4-67.2$ & $\mathrm{~A}, \mathrm{~B}, \mathrm{C}, \mathrm{L}$ & 715.4254 & 715.4277 & 3.2 & $\begin{array}{c}715.4250(80), 697.4150(12), 349.2108(4), 331.1936(100), 314.1679(12) \\
278.1462(32), 250.1514(24), 175.1165(9), 116.0691(22), 91.0531(16)\end{array}$ & $\begin{array}{l}\text { 3-(N-suberoyl argininyl) Gamabufotalin } \\
\qquad \mathrm{C}_{38} \mathrm{H}_{59} \mathrm{~N}_{4} \mathrm{O}_{9}^{+}[28]\end{array}$ \\
\hline XI & $66.5-66.7$ & B, L & 415.2090 & 415.2115 & 6.0 & $\begin{array}{c}415.2054 \text { (100), } 397.1832 \text { (3), } 333.1887 \text { (3), } 283.1621 \text { (5), } 253.0760 \text { (5), } 175.0999 \\
\text { (6) }\end{array}$ & $\begin{array}{l}\text { 19-Oxo-desacetylcinobufagin } \\
\qquad \mathrm{C}_{24} \mathrm{H}_{31} \mathrm{O}_{6}{ }^{+}[28]\end{array}$ \\
\hline 28 & $66.5-69.5$ & $\mathrm{~A}, \mathrm{~B}, \mathrm{C}, \mathrm{L}$ & 697.3766 & 697.3807 & 5.9 & $\begin{array}{c}697.3745(100), 679.3595 \text { (2), } 363.1933(3), 335.1966(4), 317.1759(61), 264.1290 \\
\text { (33), } 236.1349(11), 175.1154(4),\end{array}$ & $\begin{array}{l}\text { 3-(N-pimeloyl argininyl) Resibufagin } \\
\qquad \mathrm{C}_{37} \mathrm{H}_{53} \mathrm{~N}_{4} \mathrm{O}_{9}^{+}[28]\end{array}$ \\
\hline 18 & $68.6-72.0$ & $\mathrm{~A}, \mathrm{~B}, \mathrm{C}, \mathrm{L}$ & 715.4265 & 715.4277 & 1.7 & $\begin{array}{c}715.4266(44), 697.4156(7), 349.2136(4), 331.1944(100), 314.1708(3), 278.1484 \\
(8), 250.1538(5), 175.1193(4), 105.0695(11), 91.0540(11)\end{array}$ & $\begin{array}{l}\text { 3-(N-suberoyl argininyl) Telocinobufagin } \\
\qquad \mathrm{C}_{38} \mathrm{H}_{59} \mathrm{~N}_{4} \mathrm{O}_{9}^{+}[28]\end{array}$ \\
\hline 32 & $70.0-72.6$ & $\mathrm{~A}, \mathrm{~B}, \mathrm{C}, \mathrm{L}$ & 713.4101 & 713.4120 & 2.6 & $\begin{array}{c}713.4100(48), 695.3992(5), 365.2075(3), 331.1941(100), 314.1681(5), 296.1581 \\
(3), 278.1473(12), 250.1533(8), 175.1198(8), 112.0873(16), 91.0536(15) \\
399.2150(<1) 381.2104(24), 363.2394(14) 331.1949(35) .275 .1786(19)\end{array}$ & $\begin{array}{l}\text { 3-(N-suberoyl argininyl) Marinobufagin } \\
\qquad \mathrm{C}_{38} \mathrm{H}_{57} \mathrm{~N}_{4} \mathrm{O}_{9}+[28]\end{array}$ \\
\hline$x$ & $71.8-72.6$ & $\mathrm{~A}, \mathrm{~B}, \mathrm{C}, \mathrm{L}$ & 399.2150 & 399.2166 & 4.0 & $\begin{array}{c}239.1741 \text { (52), } 213.1297(33), 128.0661(46), 115.0554(42), 105.0684(67), \\
91.0533(100)\end{array}$ & Resibufagin $\mathrm{C}_{24} \mathrm{H}_{31} \mathrm{O}_{5}{ }^{+}[32]$ \\
\hline 29 & $74.6-78.9$ & $\mathrm{~A}, \mathrm{~B}, \mathrm{C}, \mathrm{L}$ & 711.3941 & 711.3964 & 1.1 & $\begin{aligned} & 711.3956(100), 363.1933 \text { (3), } 331.1937 \text { (40), } 278.1463 \text { (24), } 250.1522 \text { (18), } \\
& 175.1179(14)\end{aligned}$ & $\begin{array}{l}\text { 3-(N-suberoyl argininyl) Resibufagin } \\
\qquad \mathrm{C}_{38} \mathrm{H}_{55} \mathrm{~N}_{4} \mathrm{O}_{9}^{+}[28]\end{array}$ \\
\hline 7 & $74.6-75.8$ & $\mathrm{~A}, \mathrm{~B}, \mathrm{~L}$ & 671.4014 & 671.4014 & 0.0 & $\begin{array}{c}671.4048(64), 653.3916(8), 351.2269(5), 303.1638(100), 286.1403(6), 250.1151 \\
(18), 175.1213(3), 158.0926(4), 105.0691(13), 91.0525(14)\end{array}$ & $\begin{array}{l}\text { 3-(N-adipoyl argininyl) bufalin } \\
\mathrm{C}_{36} \mathrm{H}_{55} \mathrm{~N}_{4} \mathrm{O}_{8}^{+}[28]\end{array}$ \\
\hline III & $75.1-76.0$ & $\mathrm{~A}, \mathrm{~B}, \mathrm{C}, \mathrm{L}$ & 403.2464 & 403.2479 & 3.7 & $\begin{array}{c}403.2456(57), 385.2353(37), 367.2252(44), 349.2135(79), 253.1929(31), \\
215.1780(30), 105.0700(79), 91.0537(100)\end{array}$ & Telocinobufagin $\mathrm{C}_{24} \mathrm{H}_{35} \mathrm{O}_{5}{ }^{+}[29,31]$ \\
\hline 35 & $76.4-77.5$ & A, B, L & 727.3922 & 727.3913 & 4.0 & $\begin{array}{c}727.3917(100), 711.3898(6), 397.1995(9), 331.1962(100), 278.1494(35), \\
250.1532(29), 175.1180(21), 112.0885(34), 91.0540(23)\end{array}$ & $\begin{array}{l}\text { 3-(N-suberoyl argininyl) Bufotalinin } \\
\qquad \mathrm{C}_{38} \mathrm{H}_{55} \mathrm{~N}_{4} \mathrm{O}_{10}{ }^{+}[28]\end{array}$ \\
\hline IX & $78.3-79.2$ & A, B, L & 401.2323 & 401.2323 & 0.0 & $\begin{array}{c}401.2335(23), 383.2235(10), 365.2105(51), 347.2012(32), 337.2162(17), \\
319.2068(19), 269.1890(20), 253.1938(44), 251.1797(31), 239.1061(22), \\
183.1157(25), 128.0639(74), 105.0710(81), 91.0543(100)\end{array}$ & Marinobufagin $\mathrm{C}_{24} \mathrm{H}_{33} \mathrm{O}_{5}{ }^{+}$[32] \\
\hline
\end{tabular}


Table 1. Cont

\begin{tabular}{|c|c|c|c|c|c|c|c|}
\hline $\mathbf{N}^{\circ}$ & Rt (min) & Sample & $\begin{array}{l}{[\mathrm{M}+\mathrm{H}]^{+}} \\
\text {Detected }\end{array}$ & $\begin{array}{l}{[\mathrm{M}+\mathrm{H}]^{+}} \\
\text {Expected }\end{array}$ & $\begin{array}{l}\text { Error } \\
(\mathrm{ppm})\end{array}$ & MS/MS Fragmentation & Tentative Identification \\
\hline 25 & $82.6-86.3$ & A, B, C, L & 669.3840 & 669.3858 & 2.7 & $\begin{array}{c}669.3835(100), 349.2124(2), 303.1628(79), 286.1395(5), 268.1264(14), \\
250.1156(24), 175.1188(8), 116.0695(11), 91.0531(6)\end{array}$ & $\begin{array}{l}\text { 3-(N-adipoyl argininyl) Resibufogenin } \\
\qquad \mathrm{C}_{36} \mathrm{H}_{53} \mathrm{~N}_{4} \mathrm{O}_{8}^{+}[28]\end{array}$ \\
\hline 8 & $83.0-86.2$ & A, L & 685.4142 & 685.4171 & 4.2 & $\begin{array}{c}685.4113(94), 667.4030(11), 351.2260(7), 317.1779(100), 300.1539(10), \\
264.1298(24), 175.1181(9), 112.0860(22), 91.0520(20)\end{array}$ & $\begin{array}{l}\text { 3-(N-pimeloyl argininyl) bufalin } \\
\qquad \mathrm{C}_{37} \mathrm{H}_{57} \mathrm{~N}_{4} \mathrm{O}_{8}^{+}[28]\end{array}$ \\
\hline I & $87.8-88.7$ & $\mathrm{~A}, \mathrm{~B}, \mathrm{C}, \mathrm{L}$ & 387.2512 & 387.2530 & 4.6 & $\begin{array}{l}387.2482(60), 369.2396(72), 351.2291(52), 333.2204(10), 255.2101(57), \\
187.1454(14), 173.1330(16), 128.0637(36), 105.0698(88) .91 .0541(100)\end{array}$ & Bufalin $\mathrm{C}_{24} \mathrm{H}_{35} \mathrm{O}_{4}^{+}[31]$ \\
\hline 26 & $91.4-94.6$ & A, L & 683.4000 & 683.4014 & 2.0 & $\begin{array}{c}683.4015(100), 665.3974(2), 349.2114(5), 317.1791(83), 300.1531(11), \\
282.1435(11), 264.1338(25), 236.1358(13), 175.1163(10), 143.0834(6), \\
112.0868(16), 91.0546(12) .\end{array}$ & $\begin{array}{l}\text { 3-(N-pimeloyl argininyl) Resibufogenin } \\
\qquad \mathrm{C}_{37} \mathrm{H}_{55} \mathrm{~N}_{4} \mathrm{O}_{8}^{+}\end{array}$ \\
\hline 9 & $91.4-95.1$ & A, B, C, L & 699.4313 & 699.4327 & 2.0 & $\begin{array}{c}699.4311 \text { (100), } 681.4213(14), 351.2279(6), 331.1942 \text { (78), } 314.16814(9) \\
296.1568 \text { (6), } 278.1477(21), 250.1522(14), 175.1169(9), 112.0863(21), 91.0541 \\
\text { (15) }\end{array}$ & $\begin{array}{l}\text { 3-(N-suberoyl argininyl) bufalin } \\
\qquad \mathrm{C}_{38} \mathrm{H}_{59} \mathrm{~N}_{4} \mathrm{O}_{8}^{+}\end{array}$ \\
\hline VII & $97.3-98.3$ & $\mathrm{~A}, \mathrm{~B}, \mathrm{C}, \mathrm{L}$ & 385.2328 & 385.2373 & 7.7 & $\begin{array}{l}385.2318(8), 367.2221(15), 349.2133(12), 321.2151(6), 253.1923(19), 241.1195 \\
(14), 205.0860(16), 185.0928(4), 115.0544(49), 105.0689(69), 91.0531(100)\end{array}$ & Resibufogenin $\mathrm{C}_{24} \mathrm{H}_{33} \mathrm{O}_{4}{ }^{+}[29,32]$ \\
\hline 27 & 98.7-103.1 & $\mathrm{A}, \mathrm{B}, \mathrm{C}, \mathrm{L}$ & 697.4117 & 697.4171 & 7.7 & $\begin{array}{c}697.4110(100), 679.4003(2), 349.2108(3), 331.1917(85), 314.1667(7), 278.1455 \\
(26), 250.1503(19), 175.1157(9), 112.0854(19), 910525(11)\end{array}$ & $\begin{array}{l}\text { 3-(N-suberoyl argininyl) Resibufogenin } \\
\qquad \mathrm{C}_{38} \mathrm{H}_{57} \mathrm{~N}_{4} \mathrm{O}_{8}^{+}[28]\end{array}$ \\
\hline
\end{tabular}


<smiles>CCC(CCCNC(=N)N)NC(=O)NC(=O)O</smiles>

\section{Argininyl diacid derivatives}

Compd $n \quad$ Diacid

14 Adipic acid

25 Pimelic acid

36 Suberic acid

47 Azelaic acid

$5 \quad 8 \quad$ Sebacic acid

69 Undecadienoic acid

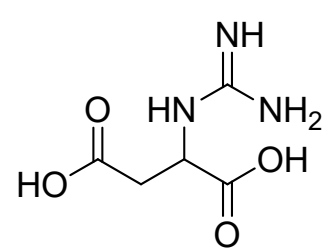

A1 Guanidinosuccinic acid

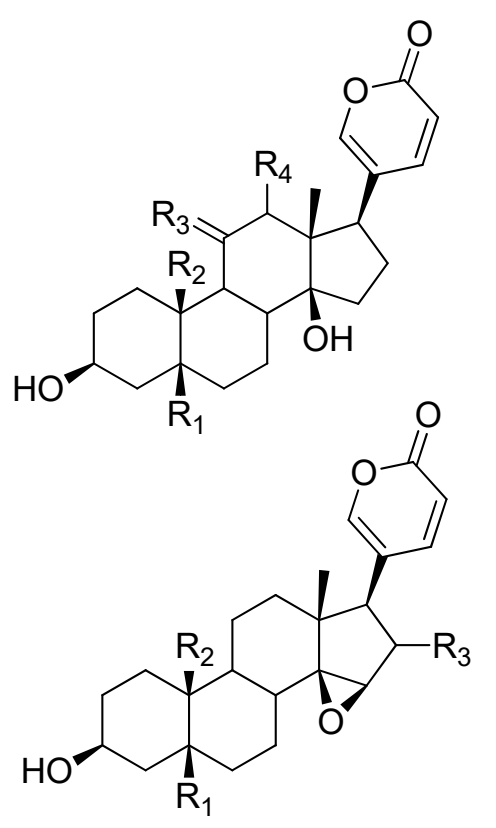

I $\mathrm{R}_{1}=\mathrm{H}, \mathrm{R}_{2}=\mathrm{CH}_{3}, \mathrm{R}_{3}=\mathrm{H}_{2}, \mathrm{R}_{4}=\mathrm{H}$

II $\mathrm{R}_{1}=\mathrm{H}, \mathrm{R}_{2}=\mathrm{CH}_{3}, \mathrm{R}_{3}=\mathrm{OH}, \mathrm{H} ; \mathrm{R}_{4}=\mathrm{H} \quad$ Gamabufotalin

III $\mathrm{R}_{1}=\mathrm{OH}, \mathrm{R}_{2}=\mathrm{CH}_{3}, \mathrm{R}_{3}=\mathrm{H}_{2}, \mathrm{R}_{4}=\mathrm{H} \quad$ Telocinobufagin

IV $\mathrm{R}_{1}=\mathrm{H}, \mathrm{R}_{2}=\mathrm{CH}_{3}, \mathrm{R}_{3}=\mathrm{H}_{2}, \mathrm{R}_{4}=\beta \mathrm{OH} \quad$ Bufarenogin

V $\mathrm{R}_{1}=\mathrm{H}, \mathrm{R}_{2}=\mathrm{CH}_{3}, \mathrm{R}_{3}=\mathrm{H}_{2}, \mathrm{R}_{4}=\alpha \mathrm{OH} \quad \psi$-Bufarenogin

VI $\mathrm{R}_{1}=\mathrm{OH}, \mathrm{R}_{2}=\mathrm{CH}_{2} \mathrm{OH}, \mathrm{R}_{3}=\mathrm{H}_{2}, \mathrm{R}_{4}=\mathrm{H}$ Hellebrigenol

XIV $\mathrm{R}_{1}=\mathrm{OH}, \mathrm{R}_{2}=\mathrm{CHO}, \mathrm{R}_{3}=\mathrm{H}_{2}, \mathrm{R}_{4}=\mathrm{H} \quad$ Hellebrigenin

VII $\mathrm{R}_{1}=\mathrm{H}, \mathrm{R}_{2}=\mathrm{CH}_{3}, \mathrm{R}_{3}=\mathrm{H} \quad$ Resibufogenin

VIII $\mathrm{R}_{1}=\mathrm{H}, \mathrm{R}_{2}=\mathrm{CH}_{2} \mathrm{OH}, \mathrm{R}_{3}=\mathrm{H} \quad$ Resibufaginol

IX $\mathrm{R}_{1}=\mathrm{OH}, \mathrm{R}_{2}=\mathrm{CH}_{3}, \mathrm{R}_{3}=\mathrm{H} \quad$ Marinobufagin

X $\quad \mathrm{R}_{1}=\mathrm{H}, \mathrm{R}_{2}=\mathrm{CHO}, \mathrm{R}_{3}=\mathrm{H} \quad$ Resibufagin

XI $\mathrm{R}_{1}=\mathrm{H}, \mathrm{R}_{2}=\mathrm{CHO}, \mathrm{R}_{3}=\mathrm{OH} \quad$ 19-Oxo-desacetylcinobufagin

XII $\mathrm{R}_{1}=\mathrm{OH}, \mathrm{R}_{2}=\mathrm{CHO}, \mathrm{R}_{3}=\mathrm{H} \quad$ Bufotalinin

XV $\mathrm{R}_{1}=\mathrm{OH}, \mathrm{R}_{2}=\mathrm{CH}_{3}, \mathrm{R}_{3}=\mathrm{OH} \quad$ Desacetylcinobufotalin

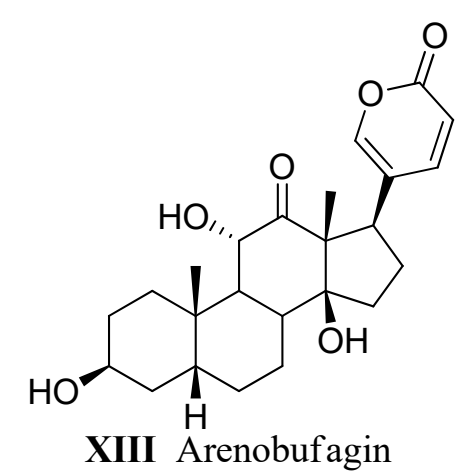

Figure 3. Structure of the compounds A1, A2, genines (bufadienolides) I-XV, and argininyl diacids 1-6 tentatively identified in PGS of R. horribilis. 


\subsubsection{Alkaloids and Guanidine Derivatives}

The alkaloid guanidinosuccinic acid (A1) and dehydrobufotenine (A2) were detected in the PGS (Figure 3). The fragmentation pattern and errors comparing the detected and expected mass fully support the identification. Dehydrobufotenin was reported in the PGS of $R$. marina and R. schneideri $[9,18]$.

\subsubsection{Bufadienolides}

The bufadienolides bufalin (I), gamabufotalin (II), telocinobufagin (III), bufarenogin/ $\psi$-bufarenogin $(\mathrm{IV}, \mathrm{V})$, hellebrigenol (VI), resibufogenin (VII), resibufaginol (VIII), marinobufagin (IX), resibufagin (X), 19-oxo-desacetylcinobufagin (XI), bufotalinin (XII), arenobufagin (XIII), and hellebrigenin (XIV) were identified by comparing the molecular formula and fragmentation patterns with literature. According to Ye and Guo [29], resibufaginol (VIII) elutes before marinobufagin (IX) and, the elution sequence agrees with our assignment (Figure 3).

\subsubsection{Argininyl Diacids of Bufadienolides}

In the toad secretions, bufadienolides occur as free and as argininyl diacid derivatives. The identity of the bufadienolide moiety followed from the analysis of the neutral loss of water from the $[\mathrm{M}+\mathrm{H}]^{+}$ion and subsequent fragmentation leading to the arginine on at $m / z 175$. The neutral loss of 368 amu from the $[\mathrm{M}+\mathrm{H}]^{+}$ion was used to identify the homologous series of bufalin derivatives. Compounds 7, 8, and 9 were identified as 3-(N-adipoylargininyl)-, 3-( $N$-pimeloylargininyl)- and 3-( $N$-suberoylargininyl) bufalin, respectively, in agreement with the literature [9]. The telocinobufagin derivatives, with an additional hydroxyl function at C-5, showed a constant neutral loss of $384 \mathrm{amu}$, as can be observed for the mass spectra of compounds 16-18. While the identity of the argininyl diacid moiety can be unambiguously assigned, telocinobufagin and its isomer gamabufotalin, with a hydroxyl function at C-11 instead of C-5, show the same molecular formula and fragments. In the $R$. horribilis poison secretion, two compounds with the molecular formula $\mathrm{C}_{36} \mathrm{H}_{54} \mathrm{~N}_{4} \mathrm{O}_{9}$ were detected at $\mathrm{R}_{\mathrm{t}} 49.8-50.1$ and 57.9-58.2 min; two products with the molecular formula $\mathrm{C}_{37} \mathrm{H}_{56} \mathrm{~N}_{4} \mathrm{O}_{9}$ at $\mathrm{R}_{\mathrm{t}}$ 53.3-54.1 and 62.4-64.7 min; and two compounds presenting the molecular formula $\mathrm{C}_{38} \mathrm{H}_{58} \mathrm{~N}_{4} \mathrm{O}_{9}$ at $\mathrm{R}_{\mathrm{t}}$ 66.4-67.2 and 68.6-72.0 min, and were assigned to the adipoyl- (compound 10 and 16), pimeloyl- (11 and 17) and suberoyl- (compounds 12 and 18) esters of gamabufotalin and telocinobufagin, respectively. According to Cao et al. [28] for argininyl diacids from gamabufotalin and telocinobufagin, the homologous series have higher Rt for the telocinobufagin than for the gamabufotalin argininyl diesters. Therefore, in the pairs with the same molecular formula and fragments, the compound with lower Rt was tentatively identified as the gamabufotalin argininyl ester. The same observation regarding Rt values is found for the genines by Ye and Guo [29] and Ren et al. [33]. Three compounds with molecular formula $\mathrm{C}_{38} \mathrm{H}_{58} \mathrm{~N}_{4} \mathrm{O}_{10}$ eluted at $\mathrm{R}_{t}$ 49.1-49.2, 54.2-54.6 and 57.0-57.8 min and fragmented to the base peak at $\mathrm{m} / \mathrm{z} 331$, in agreement with 3-(N-suberoyl argininyl) hellebrigenol (compound 19). The three compounds differ in the intensity of the ions after fragmentation and were assigned as suberoyl argininyl hellebrigenol isomers 1, 2, and 3, respectively (Figure 4).

Compounds 20-22 are isomers of the compounds 13-15, differing in the placement of the hydroxy function (C-5 for 20-22, C-11 for 13, and C-5/C-16 for 14-15). The compounds 14 and 15 present an epoxy function while there is a carbonyl ketone at C-12 in $\mathbf{1 3}$ and the aldehyde from hellebrigenin in compounds 20-22. The compounds 20-22 were assigned as adipoyl-, pimeloyl- and suberoylargininyl hellebrigenin based on the neutral loss of $398 \mathrm{amu}$, leading to the peak of the argininyl diacids. 3-(N-suberoylargininyl) hellebrigenin (hellebritoxin) was reported from the PGS of R. marina from the Peruvian Amazon [18] and Paraguayan Rhinella species [9]. 


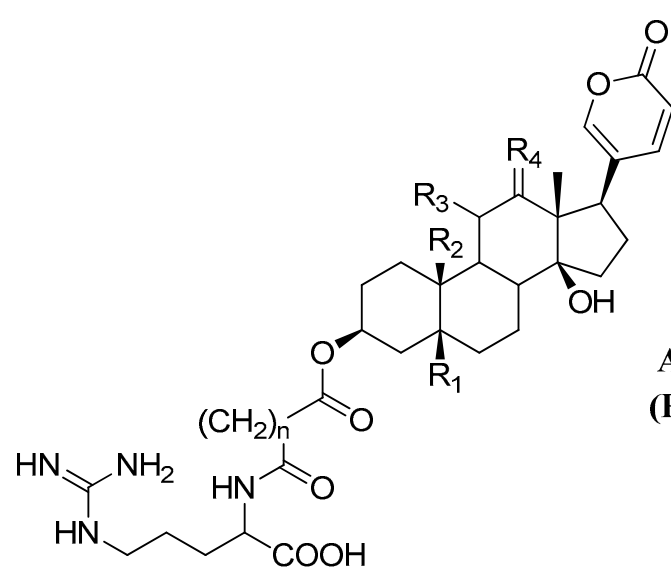

Bufalin argininyl diacids $\left(\mathbf{R}_{1}=\mathbf{H}, \mathbf{R}_{2}=\mathbf{C H}_{3}, \mathbf{R}_{3}=\mathbf{H}, \mathbf{R}_{\mathbf{4}}=\mathbf{H}_{2}\right)$

Compd $n$ Diacid

$74 \quad 4$ Adipic acid

85 Pimelic acid

96 Suberic acid

Arenobufagin argininyl diacids

$\left(\mathbf{R}_{1}=\mathbf{H}, \mathbf{R}_{2}=\mathbf{C H}_{3}, \mathbf{R}_{3}=\mathbf{O H}, \mathbf{R}_{\mathbf{4}}=\mathbf{O}\right)$

Compd $n$ Diacid

134 Adipic acid
Gamabuf otalin argininyl diacids $\left(\mathbf{R}_{1}=\mathbf{H}, \mathbf{R}_{2}=\mathbf{C H}_{3}, \mathbf{R}_{3}=\mathrm{OH}, \mathbf{R}_{\mathbf{4}}=\mathbf{H}_{2}\right)$

Compd $n$ Diacid

104 Adipic acid

115 Pimelic acid

126 Suberic acid

Hellebrigenol argininyl diacids $\left(\mathbf{R}_{1}=\mathrm{OH}, \mathbf{R}_{\mathbf{2}}=\mathrm{CH}_{\mathbf{2}} \mathrm{OH}, \mathrm{R}_{3}=\mathbf{H}, \mathbf{R}_{\mathbf{4}}=\mathrm{H}_{2}\right.$ )

Compd $n$ Diacid

196 Suberic acid
Telocinobufagin argininyl diacids $\left(\mathbf{R}_{1}=\mathrm{OH}, \mathbf{R}_{2}=\mathrm{CH}_{3}, \mathbf{R}_{3}=\mathbf{H}, \mathbf{R}_{\mathbf{4}}=\mathrm{H}_{2}\right)$

Compd $n$ Diacid

164 Adipic acid

175 Pimelic acid

1866 Suberic acid

Hellebrigenin argininyl diacids

$\left(\mathbf{R}_{1}=\mathbf{O H}, \mathbf{R}_{2}=\mathbf{C H O}, \mathbf{R}_{3}=\mathbf{H}, \mathbf{R}_{\mathbf{4}}=\mathrm{H}_{2}\right)$

$\begin{array}{lll}\text { Compd } & n & \text { Diacid } \\ \mathbf{2 0} & 4 & \text { Adipic acid } \\ \mathbf{2 1} & 5 & \text { Pimelic acid } \\ \mathbf{2 2} & 6 & \text { Suberic acid }\end{array}$

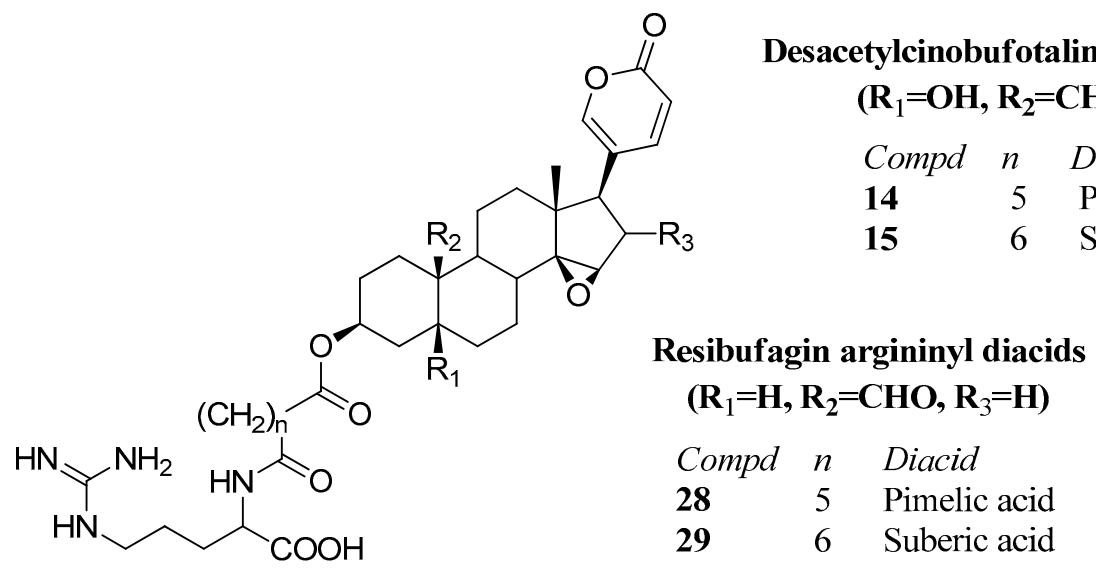

Resibufogenin argininyl diacids

$\left(\mathbf{R}_{1}=\mathbf{H}, \mathbf{R}_{\mathbf{2}}=\mathbf{C H}_{3}, \mathbf{R}_{3}=\mathbf{H}\right)$

Compd $n$ Diacid

254 Adipic acid

265 Pimelic acid

$27 \quad 6 \quad$ Suberic acid

Resibufagin argininyl diacids Marinobuf agin argininyl diacids

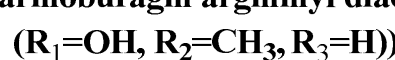

$\begin{array}{lll}\text { Compd } & n & \text { Diacid } \\ \mathbf{3 0} & 4 & \text { Adipic acid } \\ \mathbf{3 1} & 5 & \text { Pimelic acid } \\ \mathbf{3 2} & 6 & \text { Suberic acid }\end{array}$
Bufotalinin argininyl diacids $\left(\mathbf{R}_{1}=\mathbf{O H}, \mathbf{R}_{\mathbf{2}}=\mathbf{C H O}, \mathbf{R}_{3}=\mathbf{H}\right)$ Compd $n$ Diacid

$345 \quad 5 \quad$ Pimelic acid

356 Suberic acid

Figure 4. Structure of the compounds 7-22, 25-32, and 34-35 tentatively identified in the parotoid gland secretion of $R$. horribilis. 
The occurrence of hellebrigenin and resibufagin argininyl diacids in our samples was suggested by the ${ }^{1} \mathrm{HNMR}$ analysis of the PGS and fractions. The isomer pairs with the molecular formula $\mathrm{C}_{36} \mathrm{H}_{52} \mathrm{~N}_{4} \mathrm{O}_{10}, \mathrm{C}_{37} \mathrm{H}_{54} \mathrm{~N}_{4} \mathrm{O}_{10}$, and $\mathrm{C}_{38} \mathrm{H}_{56} \mathrm{~N}_{4} \mathrm{O}_{10}$, were assigned as 3-( $\mathrm{N}$-adipoyl argininyl) arenobufagin 13 and 3-( $N$-adipoyl argininyl) hellebrigenin 20; 3-( $N$-pimeloyl argininyl) desacetylcinobufotalin 14 and 3-(N-pimeloyl argininyl) hellebrigenin 21; 3-(N-suberoyl argininyl) desacetylcinobufotalin 15 and 3-(N-suberoyl argininyl) hellebrigenin 22, respectively. The assignment of the arenobufagin and hellebrigenin argininyl diacids in our samples of PGS follows the elution sequence previously reported for the genines and argininyl esters of both compounds, with hellebrigenin isomers eluting before the corresponding arenobufagin derivatives [33]. Ren et al. [33] reported that arenobufagin elutes before hellebrigenin in their PGS samples. Furthermore, the loss of $29 \mathrm{amu}$ in the hellebrigenin derivatives was observed for compounds 20-22 in agreement with Ye and Guo [29] (Figure 4).

The compound 23 showed the neutral loss of $414 \mathrm{amu}$ and a base peak in agreement with pimeloylarginine and was assigned as 3-( $N$-pimeloyl argininyl) hydroxyhellebrigenin. Two compounds with the same molecular formula $\mathrm{C}_{38} \mathrm{H}_{56} \mathrm{~N}_{4} \mathrm{O}_{11}$ and $\mathrm{R}_{\mathrm{t}}$ 48.8-49.3 and 58.1-58.4 min occur in the PGS. The mass spectrum of the compound, described here as compound $\mathbf{2 4}$, showed the neutral loss of 414 amu, leading to the suberoylargininyl ion at $\mathrm{m} / \mathrm{z} 331$. The compound was assigned as 3-(N-suberoyl argininyl) hydroxyhellebrigenin. The placement of the additional hydroxyl function in compound 24 remains to be established, being C-12 or C-16 the most probable options. The isomers were tentatively identified as 3-( $\mathrm{N}$-suberoyl argininyl) hydroxyhellebrigenin 24 isomer 1 and 2, respectively. The resibufogenin derivatives (compounds 25, 26 and 27) were assigned based on the loss of 366 amu from the $[\mathrm{M}+\mathrm{H}]^{+}$ion, leading to the argininyl diacids and were identified as adipoyl-, pimeloy- and suberoylresibufogenin, respectively. The compounds were previously reported from the Amazonian R. marina [18] and Paraguayan Rhinella species [9] (Figures 4 and 5).

The compounds $\mathbf{2 8}, \mathbf{2 9}, \mathbf{3 4}$, and 35 were tentatively assigned by the neutral loss of the bufadienolide from the $[\mathrm{M}+\mathrm{H}]^{+}$ions, leading to the argininyl esters. The compounds $\mathbf{2 8}$ and 29 with a neutral loss of $380 \mathrm{amu}$ are compatible with the aldehyde derivatives of resibufogenin (resibufagin) and were assigned as pimeloylargininyl resibufagin 28 and suberoylargininyl resibufagin $\mathbf{2 9}$, respectively. The compounds 34 and 35 showed the neutral loss of 396 amu in agreement with aldehyde derivatives of marinobufagin (bufotalinin), being assigned as pimeloylargininyl bufotalinin 34 and suberoylargininyl bufotalinin 35, respectively. The compounds 30, 31, and 32 showed the neutral loss of $382 \mathrm{amu}$, with base peak for adipoyl-, pimeloyl-, and suberoylarginine and were identified as marinobufagin argininyl diacids, in full agreement with spectrometric data reported for South American Rhinella species $[10,18]$. Marinobufagin is the 5-hydroxy derivative of resibufogenin. The compound 33, with the molecular formula $\mathrm{C}_{36} \mathrm{H}_{50} \mathrm{~N}_{4} \mathrm{O}_{9}$ shows one additional unsaturation than compound 30 , it presents the neutral loss of $380 \mathrm{amu}$ and the base peak for adipoyl arginine. It was tentatively assigned as 3 -( $\mathrm{N}$-adipoyl argininyl) marinobufagin-9,11-ene 33. Two compounds with the molecular formula $\mathrm{C}_{38} \mathrm{H}_{54} \mathrm{~N}_{4} \mathrm{O}_{11}$ eluted at $\mathrm{R}_{\mathrm{t}}$ 52.1-52.4 and 54.7-54.9 min, respectively, and showed the neutral loss of $412 \mathrm{amu}$, suggesting the occurrence of hydroxybufotalinin derivatives differing in the placement of the hydroxyl group. For hydroxybufotalinin, the most common hydroxylation places are either at C-11, C-12, or C-16. However, we are not able to fully assign the most probable isomers. The compound $\mathbf{3 6}$ was tentatively identified as 3-( $N$-suberoyl argininyl) hydroxybufotalinin 36 isomers 1 and 2, respectively (Figures 4 and 5).

In this study, we identified a high number of constituents (55 compounds) from PGS of $R$. horribilis compared to the 29 compounds previously reported from three Peruvian Amazon populations of R. marina [18]. Although this difference might be explained by the different environments, access to food or the sensibility of the instrument used, we are not excluding the hypothesis of unique chemical composition patterns for both species. This is especially relevant if is considered that both $R$. marina and, previously confused $R$. horribilis, have geographical, genetic, and morphological differences [24,34]. The main compounds in the PGS are marinobufagin and bufalin derivatives, with aldehydes as minor constituents of the mixture. Oxidation of the methyl group at C-10 led to the primary alcohols 
and then to the corresponding aldehydes, giving origin to the bufotalinin derivatives starting from marinobufagin, resibufagin derivatives from resibufogenin, and hellebrigenin from telocinobufagin, respectively. Further oxidation of the steroid moiety led to alcohol and ketone functions mainly at C-11, C-12, or C-16. The unambiguous identification of the minor constituents requires isolation by preparative HPLC and extensive spectroscopic and spectrometric studies to identify the exact position and stereochemistry of the functional groups. Some differences with the Peruvian R. marina are the occurrence of arenobufagin derivatives and a higher number of oxidized compounds in $R$. horribilis compared with the Amazonian species. Interestingly, most of the compounds reported in the $R$. horribilis PGS have been previously described from South American Rhinella species $[9,10,18]$ and/or from the crude drug Venenum Bufonis or Ch'an Su from China and Eastern Asia [27,30,31], which exhibit anti-cancer effects in several cancer cell lines [35-40]. Based on this, we propose to evaluate the anti-cancer effect of crude PGS of R. horribilis and their fractions A, B, and C.
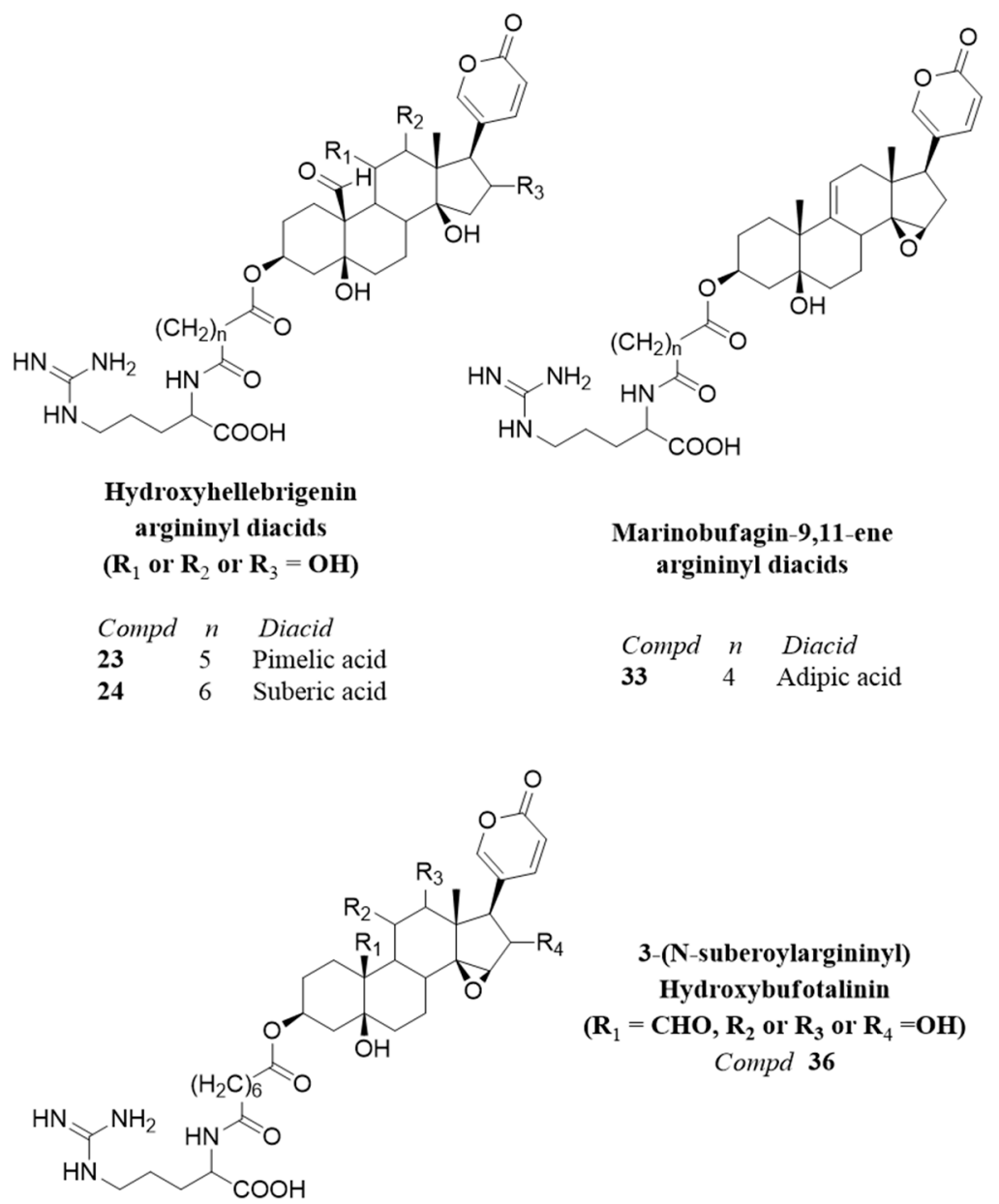

Figure 5. Structure of the compounds 23-24, 33, and 36 tentatively identified in the parotoid gland secretion of $R$. horribilis.

2.4. Effect of R. horribilis PGS on Proliferation, Clonogenic Capacity and Reactive Oxygen Species (ROS) Levels of Lung Cancer Cells

To evaluate the anti-proliferative activity of crude PGS (L) of R. horribilis and their fractions A, B, and C, lung cancer A549 cells were treated with increasing concentrations by 24 and $48 \mathrm{~h}$ and the $\mathrm{IC}_{50}$ values were calculated. As Table 2 shows, the crude PGS had an $\mathrm{IC}_{50}$ value of $0.031 \pm 0.007 \mu \mathrm{g} / \mathrm{mL}$ at $24 \mathrm{~h}$, and fractions $\mathrm{A}, \mathrm{B}$, and $\mathrm{C}$ were close to $3.08,4.4$, and 1.26 folds more active than crude PGS, respectively and this was a concentration-dependent effect (Figure $6 \mathrm{~A}$ ). At $48 \mathrm{~h}$, fraction C exhibited a strong increase in its anti-proliferative activity. 
Table 2. Anti-proliferative effect of fractions A, B, and C and crude extract of parotoid gland secretion of $R$. horribilis on lung cancer A549 cells. Data are expressed as $\mathrm{IC}_{50}$ values $(\mu \mathrm{g} / \mathrm{mL} \pm \mathrm{SEM})$ of three independent experiments.

\begin{tabular}{ccccc}
\hline Time & Crude Extract & Fraction A & Fraction B & Fraction C \\
\hline $24 \mathrm{~h}$ & $0.031 \pm 0.007$ & $0.010 \pm 0.001$ & $0.007 \pm 0.001$ & $0.025 \pm 0.001$ \\
$48 \mathrm{~h}$ & $0.015 \pm 0.001^{\mathrm{a}}$ & $0.011 \pm 0.003$ & $0.006 \pm 0.001$ & $0.007 \pm 0.002^{\mathrm{a}}$ \\
\hline \multicolumn{4}{c}{ a Significant difference compared to the same fraction $(t$ - }
\end{tabular}

${ }^{a}$ Significant difference compared to the same fraction ( $t$-test two-tailed, IC 95\%).

A

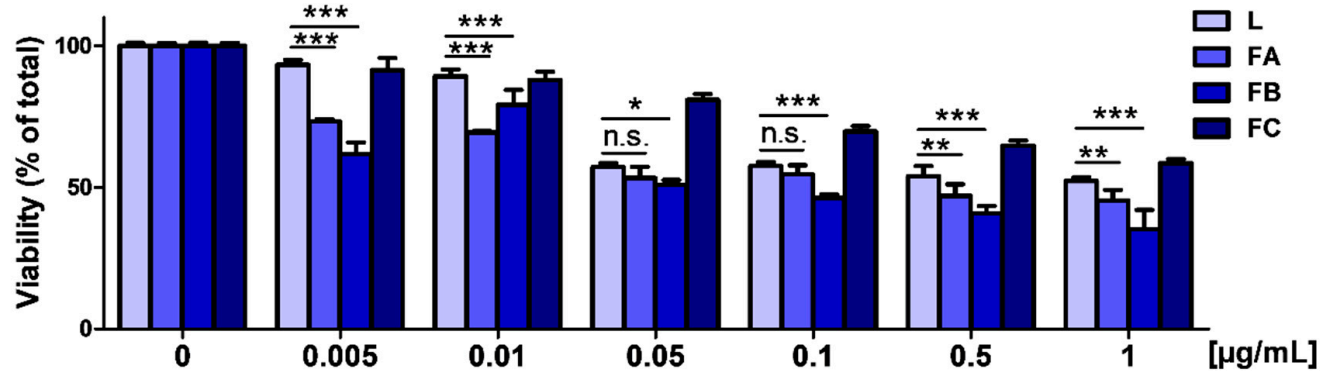

B

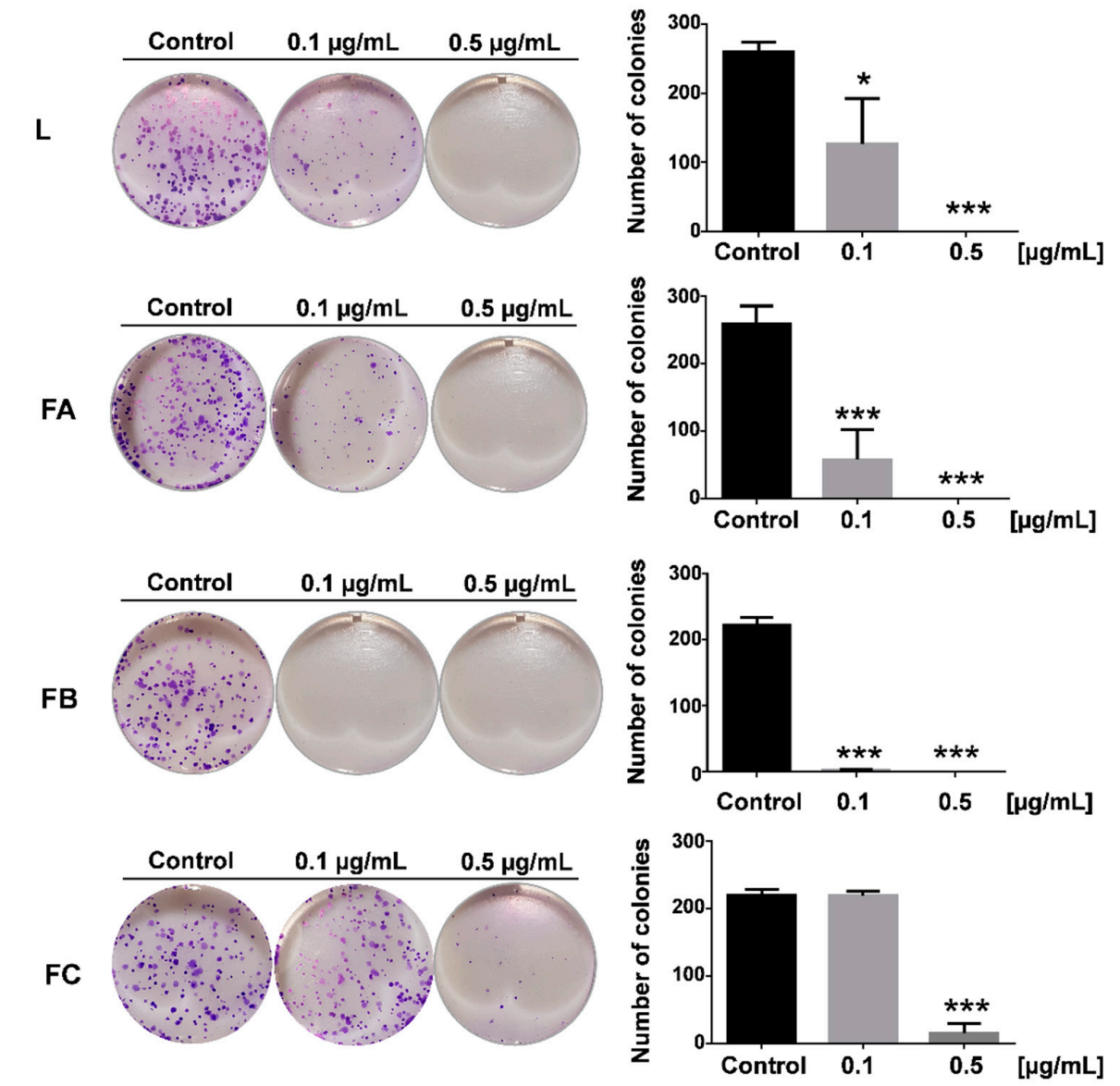

Figure 6. Effect of fractions A, B, C (FA, FB, FC) and crude extract (L) of parotoid gland secretion (PGS) of $R$. horribilis on viability and clonogenic potential of lung cancer A549 cells. (A) Effect of fractions and crude extract on viability at $24 \mathrm{~h}$ of exposure. (B) Cells were exposed $24 \mathrm{~h}$ to DMSO (control), crude extract $(\mathrm{L})$, and fractions (A, B, and C); then, the culture medium was removed and replaced by fresh medium. The number of colonies was determined at 7 days, using crystal violet staining. Data shown are the mean \pm SEM of three independent experiments. ${ }^{*} p<0.05,{ }^{* *} p<0.01,{ }^{* * *} p<0.001$, vs. control (DMSO); n.s., not significant. 
The colony formation is the ability of a single cancer cell to proliferate indefinitely, growing into a colony [41]. Since that the concentrations 0.1 and $0.5 \mu \mathrm{g} / \mathrm{mL}$ shown significant differences in viability (Figure 6A), we evaluate if the crude PGS and theirs fraction A, B, and C irreversibly affect the clonogenic potential of A549 cancer cells. These cells were treated for $24 \mathrm{~h}$ with 0.1 and $0.5 \mu \mathrm{g} / \mathrm{mL}$ of crude PGS and their fractions and then, the culture medium was washed and replaced by a fresh and PGS-free culture medium for seven days. As Figure 6B shows, fraction B evoked a strong and irreversible inhibition of the clonogenic potential and fraction $C$ was only active at $0.5 \mu \mathrm{g} / \mathrm{mL}$. Moreover, we evaluate the effect of the $R$. horribilis poison secretion and their fractions on reactive oxygen species (ROS) levels in A549 cancer cells. As Figure 7 shows, all the conditions produced a significant increase in the intracellular ROS levels. Taken together, these results suggest that the PGS of R. horribilis increases ROS production and inhibits the proliferation and clonogenic capacity of lung cancer cells, being the fraction $\mathrm{B}$ the most active.

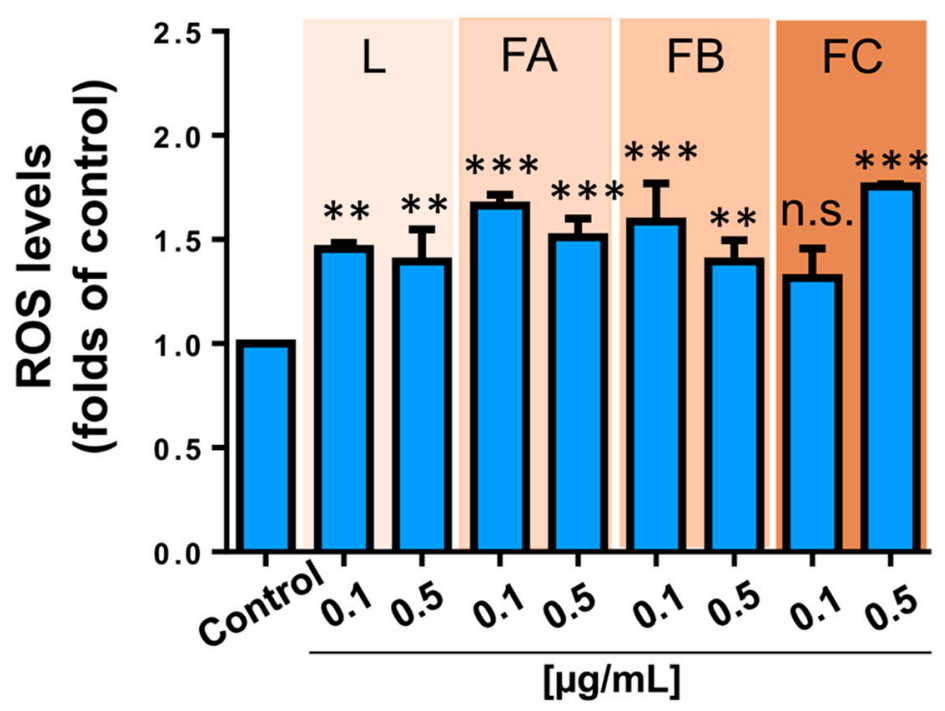

Figure 7. Effect of fractions and crude extract of parotoid gland secretions of $R$. horribilis on ROS production in A549 cancer cells. Cells were exposed $24 \mathrm{~h}$ to DMSO (control), crude extract (L), and fractions (A, B, and C), and changes in the ROS levels were determined using dihydroethidium (DHE) probe by flow cytometry. Data shown are the mean \pm SEM of three independent experiments. ** $p<0.01,{ }^{* * *} p<0.001$, vs. control (DMSO). n.s. not significant.

\subsection{Effect of R. horribilis PGS and Their Fractions on Etoposide-Induced A549 Cell Death}

Etoposide (Eto) is a known topoisomerase II inhibitor used in the treatment of several solid cancer, inducing DNA damage, growth arrest, and apoptosis in a concentration-dependent manner [42]. In several cancer cell lines, Eto produces cell cycle arrest in G2-phase [43] through p53 stabilization [44], promoting apoptosis [45]. On the other hand, we have described that the poison secretions of Rhinella schneideri, R. ornata, and R. marina produce G1- or S-phase arrest $[9,18]$, avoiding the cell cycle progression to the G2-phase checkpoint. Therefore, to determine the action of poison secretions of $R$. horribilis on phases of the cell cycle, we evaluate its effect (at $0.5 \mu \mathrm{g} / \mathrm{mL}$ ) on G2/M arrest-induced cell death by Eto. We hypothesized that whether the $R$. horribilis poison secretion acts on the cell cycle in an earlier phase different to G2/M phase, it will produce a systematic decrease of Eto-induced cell death.

At $48 \mathrm{~h}$ of treatment, $25 \mu \mathrm{M}$ Eto produced $45.50 \pm 1.38 \%$ of positive annexin $\mathrm{V}$ subpopulation $(p<0.001$ vs. control), suggesting apoptotic cell death as described [46,47]. As Figure 8 shows, only fraction B induced $22.51 \pm 3.32 \%$ of apoptotic cancer cells and crude PGS and other fractions lacked cytotoxic effect. As it was hypothesized, the combination of the R. horribilis poison secretion or their fractions plus $25 \mu \mathrm{M}$ Eto prevented the apoptosis induced by the treatment of only Eto (Figure 8), 
suggesting that anti-proliferative effect of the $R$. horribilis poison secretion is produced by cell cycle blocking before of $\mathrm{G} 2 / \mathrm{M}$ phase.

A

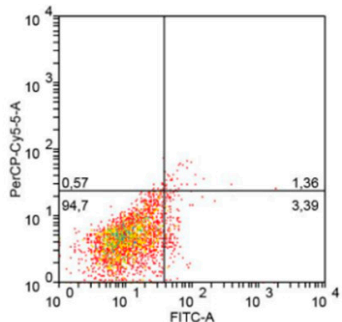

Control

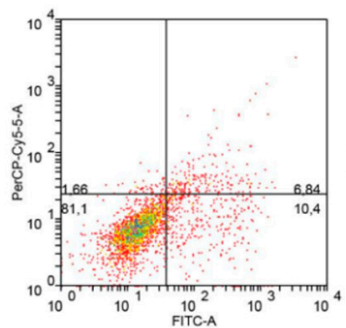

FA

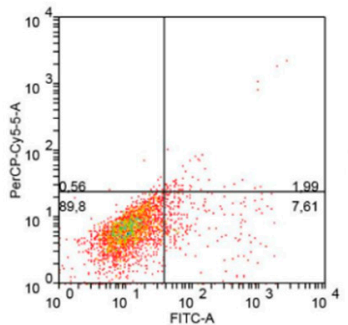

FC

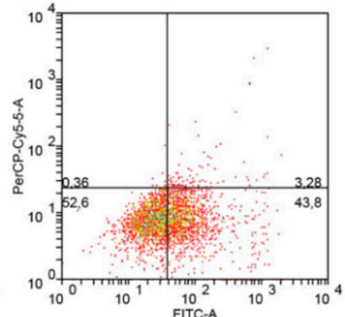

etoposide

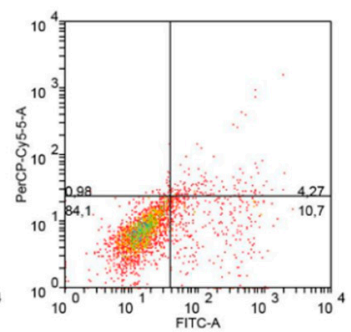

FA + etoposide

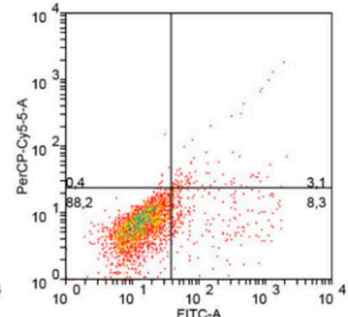

$\mathbf{L}$

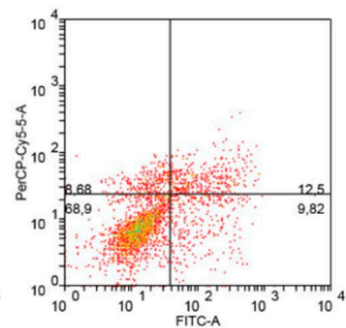

FB

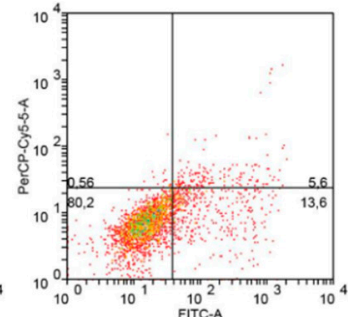

L + etoposide

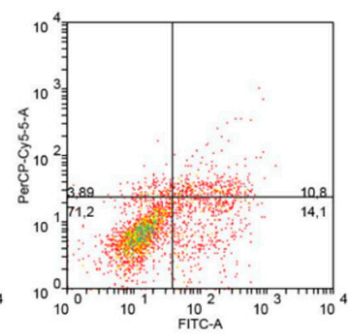

FB + etoposide

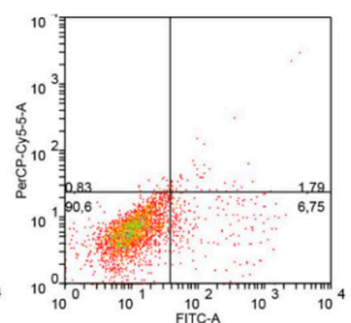

FC + etoposide

B

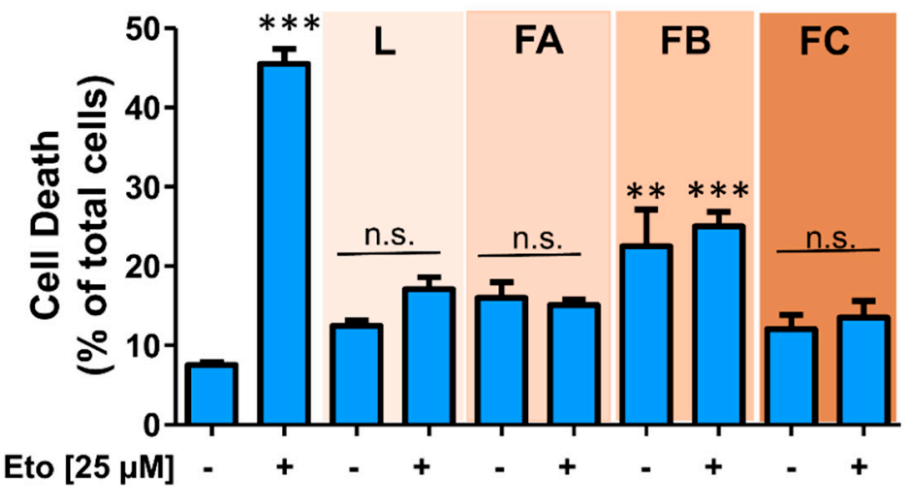

Figure 8. Effect of fractions A, B, C, and crude extract of parotoid gland secretions of $R$. horribilis on etoposide-induced cell death in A549 cancer cells. Cells were exposed 48 h to DMSO (control), crude extract (L), and fractions (FA, FB, and FC) at $0.5 \mu \mathrm{g} / \mathrm{mL}$ in the presence and absence of $25 \mu \mathrm{M}$ etoposide (Eto), and viability were determined using Annexin V/P.I. assay by flow cytometry. (A) Representative dot-plots; (B) quantification. Data shown are the mean \pm SEM of three independent experiments. ${ }^{* *} p<0.01,{ }^{* * *} p<0.001$, vs. control (DMSO). n.s. not significant.

\subsection{Effect of Fractions B and C of the R. horribilis PGS on the Migration of Lung Cancer Cells}

To evaluate the effect of components of $R$. horribilis poison secretion on the migration of cancer cells, we selected both the most active (fraction $B$ ) and the less active (fraction $C$ ) on inhibition of proliferation. The lung cancer cells were exposed to both fractions by $24 \mathrm{~h}$ and the effect on fibronectin-dependent migration was measured. At 0.1 and $0.5 \mu \mathrm{g} / \mathrm{mL}$, fraction B reduced the migrated cells to $0.79 \pm 0.03$ 
and $0.28 \pm 0.04$ folds of Control and fraction $C$ decreased the migration to $0.95 \pm 0.28$ and $0.60 \pm 0.05$ folds of Control, respectively. As Figure 9 shows, fraction B was more active inhibiting the migration of A549 cells.

Although certain bufadienolides present in the poison secretion of toads produce cell cycle arrest in S-phase [48], reduce the activity and protein levels of topoisomerases [49,50] and intercalates with DNA [51]; other DNA damage-independent mechanisms have also been recently described [8,26]. For example, the major constituents of the PGS of Rhinella species such as bufalin, marinobufagin, telocinobufagin, and hellebrigenin exhibit strong binding affinities to tubulin in vitro and in vivo conditions, producing dysregulated microtubule arrangement and G2/M cell cycle arrest in cancer cells [8]. In this work, we identified these same tubulin-inhibiting bufadienolides from $R$. horribilis poison secretions and their fractions, producing irreversible inhibition of proliferation and clonogenic capacity, increasing the ROS production, and inhibiting the fibronectin-dependent migration of human lung cancer A549 cells. An interestingly mechanistic explanation of these effects may be proposed, taking into consideration the effects of current MTA on cancer cells mentioned above [16].
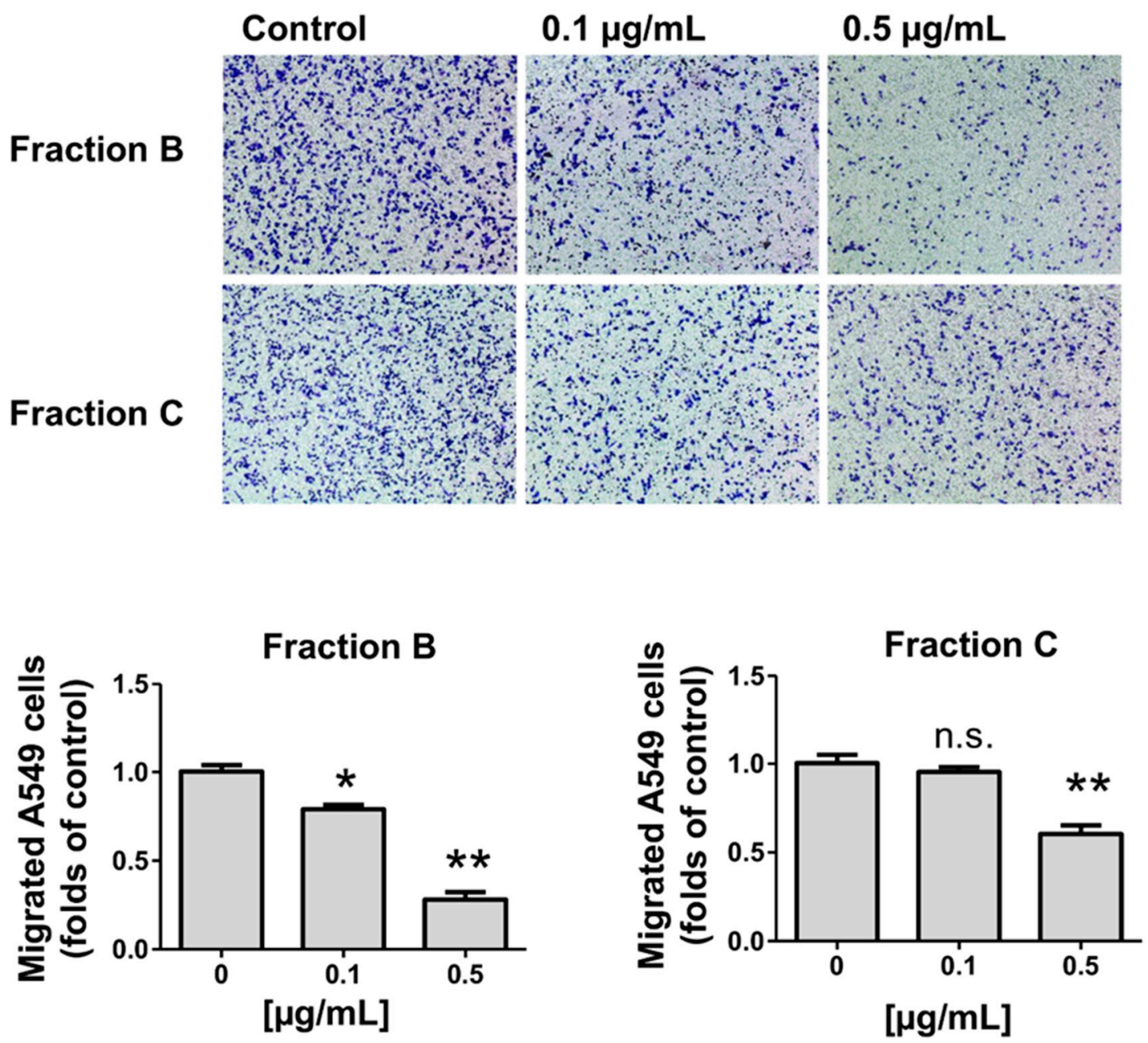

Figure 9. Effect of fractions B and C on fibronectin-dependent migration in A549 cells treated for $24 \mathrm{~h}$. Data shown are the mean \pm SEM of two independent experiments. ${ }^{*} p<0.05,{ }^{* *} p<0.01$, vs. control (DMSO). n.s. not significant.

\section{Conclusions}

The composition and biological effects of the PGS of the Amazonian R. marina has been extensively studied $[8,18,26]$, while that of $R$. horribilis remained unknown. In this study, some 55 constituents, including argininyl diacids, bufadienolides argininyl diacids, bufadienolides, and alkaloids were 
identified/tentatively identified in the PGS of $R$. horribilis for the first time. The number of constituents of this species historically confused with $R$. marina [24], was higher than the 29 compounds reported from three Peruvian Amazon populations of R. marina [18].

The alkaloids from the venoms are mainly tryptophan derivatives and some of them present anti-inflammatory effects, as shown by Zhang, et al. [52] on Chinese samples. Several studies on the composition of PGS have been undertaken with the methanol soluble fraction of the crude venom. Recently, the effect was reported of PGS of Bufo bufo and $R$. marina on metastatic melanoma, finding that the bufadienolide bufalin was the best antiproliferative agent in the samples [53]. Sinhorin, et al. [54] described the composition of the Amazonian Rhinella margaritifera PGS and reported argininyl diacids, bufotalin, and cinobufagin argininyl diacids as well as the bufadienolides bufotalin, cinobufagin, and telocinobufagin as main compounds.

The composition of the PGS in the Peruvian species $R$. horribilis differs from that of $R$. margaritifera by the higher ratio of argininyl diesters, a more complex array of oxidized bufadienolides argininyl diesters, the absence of cinobufagin, and the presence of resibufogenin. When compared with the Rhinella species from the Paraguayan river basin area, the toad from the northern Peruvian coast has a much lower content of marinobufagin and its argininyl diacids, that are the main compounds in $R$. schneideri and $R$. ornata, while cinobufagin argininyl diesters are relevant for $R$. scitula [9]. Marinobufagin and its argininyl diesters are the main compounds in $R$. marina from the Peruvian Amazon basin [18]. The chemical composition of the Peruvian coast species shows a different profile that may be associated with a different diet and genetic factors. An update on the chemistry, bioactivity, and quality control of the Asian toad Bufo gargarizans has been recently published [55] and provides relevant information to be considered for further studies on the South American species.

Although we describe the anti-cancer effect of the $R$. horribilis PGS, some issues remain to be explored, such as the action of the isolated constituents of crude secretion and the effect on non-tumoral cells and in vivo studies. The $R$. horribilis poison secretions produced irreversible inhibition of proliferation and clonogenic capacity, increasing the ROS production, and inhibiting the fibronectin-dependent migration of human lung cancer A549 cells. The fraction B was the most active on proliferative and migration of cancer cells, suggesting that certain constituents present only in this fraction as minor compounds or the combination of the many different compounds acting in synergy may be attractive candidates for further anti-cancer studies.

\section{Materials and Methods}

\subsection{Parotid Glands Secretions (PGS) Samples}

Twenty female Rhinella horribilis (Wiegmann, 1833) toads were collected in the Region of Piura, on the north coast of Peru, between December 2018 to January 2019 with herpetologists from Universidad Nacional de Trujillo. The size and weight of the animals ranged between 15.2 to $19.8 \mathrm{~cm}$ and 145 to $310 \mathrm{~g}$, respectively. The animals were gently placed in cardboard boxes and then transferred to the Pharmacognosy Laboratory of Universidad Nacional de Trujillo, Peru. The skin secretions were obtained by mild electric stimulation using platinum electrodes (4-ms pulse width, $50 \mathrm{~Hz}, 5 \mathrm{~V}$ ) (Memex) by ten seconds, as described by Thirupathi, et al. [56]. Next, the parotid glands of the toads were gently pressed with the hands to remove the PGS, which was placed in a beaker. All animals were released after collecting the samples. The fresh PGS (5.4 g) was resuspended in $\mathrm{MeOH}$ and was sonicated for $5 \mathrm{~min}$, filtered, taken to dryness under reduced pressure, and lyophilized, affording $900 \mathrm{mg}$ of the crude extract of PGS. The $w / w$ extraction yield of the $\mathrm{MeOH}$ extract was $16.7 \%$.

The obtention of peptides from the toad venom needs a different protocol than that used in our work. The venoms should be extracted with a saline buffer [22] or with acidified water and not with organic solvents. The clean-up requires methods used for protein isolation and identification. The identification of the peptides also requires different analytical tools, MALDI-TOF detection, microsequencing, and synthesis [23]. 


\subsection{Thin-Layer Chromatography Analysis}

For the thin-layer chromatography (TLC) analysis, commercial plates coated with silica gel $\mathrm{F}_{254}$ as the stationary phase were used. For visualization of the TLC profile, the plates were first observed immediately after the TLC at wavelengths ( $\lambda$ ) 254 and $365 \mathrm{~nm}$. Afterward, the plates were sprayed with a $p$-anisaldehyde solution reagent freshly prepared $(2.0 \mathrm{~mL} p$-anisaldehyde in $340 \mathrm{~mL}$ ethanol, $40 \mathrm{~mL}$ glacial acetic acid, and $20 \mathrm{~mL} 97 \%$ sulfuric acid) followed by heating up to $105^{\circ} \mathrm{C}$ for maximum visualization of spots.

\subsection{HPLC-DAD Analysis}

The system used was a Shimadzu ${ }^{\circledR}$ Prominence (Shimadzu Corporation, Kyoto, Japan) consisting of a LC-20AT pump, a SPD-M20A UV diode array detector, CTO-20 AC column oven, and a LabSolutions software (Version 5.51, Shimadzu Corporation, Kyoto, Japan). A MultoHigh 100 RP 18, 5 $\mu$ column $(250 \times 4.6 \mathrm{~mm})(\mathrm{CS}-$ Chromatographie Service $\mathrm{GmbH}$, Langerwehe, Germany) was used. The temperature was set at $30^{\circ} \mathrm{C}$. The HPLC analyses were performed using a linear gradient solvent system consisting of methanol:acetonitrile $50: 50(\mathrm{~A})$ and $0.1 \%$ formic acid in water (B) with a flow rate of $0.4 \mathrm{~mL} / \mathrm{min}$ as follows: initial conditions were $8 \% \mathrm{~A}$ and $92 \% \mathrm{~B} ; 0-10 \mathrm{~min}$ : $92 \%$ to $85 \% \mathrm{~B} ; 10-25 \mathrm{~min}$ : $85 \%$ to $70 \%$ B; $25-35 \mathrm{~min}: 70 \%$ to $65 \% \mathrm{~B} ; 35-45 \mathrm{~min}: 65 \%$ to $55 \% \mathrm{~B} ; 45-55 \mathrm{~min}$ : $55 \%$ to $50 \%$ B; 55 to $75 \mathrm{~min}$ : $50 \%$ to $45 \% \mathrm{~B} ; 75$ to $95 \mathrm{~min}: 45 \%$ to $35 \% \mathrm{~B}$; 95 to $100 \mathrm{~min}$ : $35 \%$ to $25 \% \mathrm{~B} ; 100$ to $105 \mathrm{~min}: 25 \% \mathrm{~B}$; 105 to $110 \mathrm{~min}: 25 \%$ to $40 \% \mathrm{~B} ; 110$ to $120 \mathrm{~min}$ : $40 \%$ to $60 \% \mathrm{~B} ; 120$ to $130 \mathrm{~min}: 60 \%$ to $75 \% \mathrm{~B} ; 130$ to 135 min: $75 \%$ to $92 \%$ B; 135 to 145 min: back to $92 \%$ B (initial condition). The volume injected was $20 \mu \mathrm{L}$. The compounds were monitored at 254, 280, and $330 \mathrm{~nm}$. UV spectra of the compounds detected were recorded from 200 to $500 \mathrm{~nm}$ for peak characterization.

\subsection{HPC-DAD-Q-TOF-MS/MS Analysis}

HPLC-DAD-MS/MS analysis was performed using a UHPLC/HPLC-DAD Bruker Elute LC system coupled in tandem with a Q-TOF Compact (Bruker Daltonik, Bremen, Germany). The same above-mentioned reverse phase column and a linear gradient solvent system (HPLC-DAD analysis, Section 4.3) were performed. The volume injected was $5 \mu \mathrm{L}$ of a $10 \mathrm{mg} / \mathrm{mL}$ solution.

The data were acquired in the full scan mode (range of $m / z$ 50-1500) in positive electrospray ionization mode using capillary voltage at $4.5 \mathrm{kV}$; nebulizer and drying gas $\left(\mathrm{N}_{2}\right)$ at 4.0 bar and $9.0 \mathrm{~L} / \mathrm{min}$, respectively; dry temperature of $200^{\circ} \mathrm{C}$. The MS/MS spectra were acquired in auto mode using variable collision energy of 250 to $100 \%$ of $10 \mathrm{eV}$. Internal calibration of the instrument was accomplished using $10 \mathrm{mM}$ sodium formate solution introduced to the ion source via a $20 \mu \mathrm{L}$ loop at the beginning of each analysis. The compounds were identified by comparison of the fragmentation pattern, UV profile, and mass spectra.

\subsection{NMR Analysis}

The NMR spectra were recorded on a Bruker Avance 400 spectrometer (Bruker, Rheinstetten, Germany) at $400 \mathrm{MHz}$ for ${ }^{1} \mathrm{H}$ and $100 \mathrm{MHz}$ for ${ }^{13} \mathrm{C}$ in $\mathrm{CD}_{3} \mathrm{OD}$. Chemical shifts are given in ppm with residual methanol as the internal standard.

\subsection{Cell Culture Conditions}

Human lung cancer A549 cells were grown in Dulbecco's modified Eagle's medium (DMEM), containing $25 \mathrm{mM}$ glucose and $4 \mathrm{mM}$ glutamine supplemented with $10 \%$ fetal bovine serum (FBS), penicillin $(100 \mathrm{IU} / \mathrm{mL})$, and streptomycin $(100 \mu \mathrm{g} / \mathrm{mL})$. No exogenous pyruvate supplementation was added, and cells were maintained in a humidified atmosphere at $37^{\circ} \mathrm{C}$ and $5 \% \mathrm{CO}_{2}$. 


\subsection{Colony Formation}

For the colony assay, A549 cells were seeded in 6-well plates at 500 cells per well according to Franken et al. [41] and incubated for $24 \mathrm{~h}$. The cells were treated with the crude extract (L) and fractions A, B and, C of $R$. horribilis secretions for $24 \mathrm{~h}$. After treatment, the medium was replaced with fresh medium, and the cells were incubated for 7 days to allow colony formation. Colonies were stained with $0.5 \%$ crystal violet solution in $20 \%$ methanol and washed with tap water. Colony formation was analyzed with ImageJ software (NIH, Bethesda, MD, USA) as we described [57].

\subsection{Determination of ROS Levels}

The generation of intracellular oxidative stress was determined using the dihydroethidium (DHE) probe as previously described [58]. In brief, A549 cells were grown in complete media, seeded in 12-well plates (50.000 cells/mL) allowed overnight to attach. Cells were exposed for $24 \mathrm{~h}$ to DMSO (Control) or 0.1 and $0.5 \mu \mathrm{g} / \mathrm{mL}$ of crude poison secretion (L) or A, B, and C fractions. Then, culture media was replaced by a solution containing $5 \mu \mathrm{M}$ DHE in Hank's balanced salt solution (HBSS) and incubated for $20 \mathrm{~min}$ in the dark. After this time, cells were washed, trypsinized, and resuspended in $200 \mu \mathrm{L}$ of HBSS and measured by FACSCanto flow cytometer.

\subsection{Migration Assay}

Cell migration was evaluated in Boyden Chamber assays (Transwell Costar, 6.5-mm diameter, 8- $\mu \mathrm{m}$ pore size) as previously reported [58]. The cancer cells were exposed to fractions B and C (0.1 and $0.5 \mu \mathrm{g} / \mathrm{mL})$ for $24 \mathrm{~h}$. Then, cancer cells $(50,000$ cells $/ \mathrm{mL})$ were re-suspended in serum-free medium, plated on top of the chamber insert coated with fibronectin $(2 \mu \mathrm{g} / \mathrm{mL})$, and incubated at $37^{\circ} \mathrm{C}$ for $2 \mathrm{~h}$. The inserts were removed, washed, and the bottom side of the inserts was stained with $0.5 \%$ crystal violet solution in $20 \%$ methanol. Cells from eight different frames were counted for each condition in an inverted microscope.

\subsection{Annexin V/Propidium Iodide Staining}

The cell death was evaluated using annexin V/propidium iodide (AV/PI) dual staining, following the instructions of the Annexin V-FITC Apoptosis Detection Kit (Abcam, Cambridge, UK), as previously reported [57]. In brief, A549 cancer cells were seeded (50.000 cells/mL) in 12-well plates, treated with $0.5 \mu \mathrm{g} / \mathrm{mL}$ of the crude extract (L) and fractions for $24 \mathrm{~h}$. The fluorescence was measured by flow cytometry.

\subsection{Statistics Analysis}

All statistical analyses were performed using Graph Pad Prism 4.03 (GraphPad Software, San Diego, California, USA). The data are expressed as mean \pm SEM of three independent experiments. Statistical analysis was performed using one-way or two-way ANOVA with Bonferroni's post-test for pairwise comparisons. The data were considered statistically significant when $p<0.05$.

Author Contributions: Conceptualization, G.S.-H., J.P.d.A., M.R.S.-V., P.A.A.A.-G. and F.A.U.; investigation, J.P.d.A., M.R.S.-V., P.A.A.A.-G., C.P., S.F.-R., M.M. and P.C.; writing-original draft preparation, G.S.-H. and F.A.U.; writing-review and editing, G.S.-H. and F.A.U. All authors have read and agreed to the published version of the manuscript.

Funding: This work was supported by ANID PCI-Biotechnology \#Redbio0027, FONDEQUIP EQM 170023, and "líneas de apoyo a la investigación financiadas por ICBM (2020)". J.P.de A. thanks the Universidad de Talca for a Postdoctoral grant and CP thanks ANID for Master fellowship \#22191223.

Conflicts of Interest: The authors declare no conflict of interest. 


\section{References}

1. Catenazzi, A.; Lehr, E.; May, R.V. The amphibians and reptiles of Manu National Park and its buffer zone, Amazon basin and eastern slopes of the Andes, Peru. Biota Neotrop. 2013, 13, 269-283. [CrossRef]

2. Moravec, J.; Lehr, E.; Cusi, J.C.; Córdova, J.H.; Gvoždík, V. A new species of the Rhinella margaritifera species group (Anura, Bufonidae) from the montane forest of the Selva Central, Peru. Zookeys 2014, 35-56. [CrossRef]

3. Pejic, R.; Simic Prskalo, M.; Simic, J. Ocular Hypotonia and Transient Decrease of Vision as a Consequence of Exposure to a Common Toad Poison. Case Rep. Ophthalmol. Med. 2020, 2020, 2983947. [CrossRef]

4. Isoardi, K.; Lee, S.; Burren, J.M.; George, J.; Goggin, A.; Chan, B.S. Bufadienolide toxicity in a child following cane toad egg ingestion. Emerg. Med. Australas. 2018, 30, 728-729. [CrossRef]

5. Peterson, M.E.; Roberts, B.K. Toads. In Small Animal Toxicology, 3rd ed.; Peterson, M.E., Talcott, P.A., Eds.; Elsevier: Amsterdam, The Netherlands, 2013; pp. 833-839.

6. McClelland, D. The Moche Botanical Frog. Arqueol. Iberoam. 2011, 10, 30-42. [CrossRef]

7. Donnan, C.B. Moche Art of Peru; Museum of Cultural Heritage, University of California: Los Angeles, CA, USA, 1978; p. 205.

8. Abdelfatah, S.; Lu, X.; Schmeda-Hirschmann, G.; Efferth, T. Cytotoxicity and antimitotic activity of Rhinella schneideri and Rhinella marina venoms. J. Ethnopharmacol. 2019, 242, 112049. [CrossRef] [PubMed]

9. Schmeda-Hirschmann, G.; Gomez, C.V.; Rojas de Arias, A.; Burgos-Edwards, A.; Alfonso, J.; Rolon, M.; Brusquetti, F.; Netto, F.; Urra, F.A.; Cárdenas, C. The Paraguayan Rhinella toad venom: Implications in the traditional medicine and proliferation of breast cancer cells. J. Ethnopharmacol. 2017, 199, 106-118. [CrossRef] [PubMed]

10. Schmeda-Hirschmann, G.; Quispe, C.; Theoduloz, C.; de Sousa, P.T., Jr.; Parizotto, C. Antiproliferative activity and new argininyl bufadienolide esters from the "cururú" toad Rhinella (Bufo) schneideri. J. Ethnopharmacol. 2014, 155, 1076-1085. [CrossRef] [PubMed]

11. Indini, A.; Rijavec, E.; Bareggi, C.; Grossi, F. Novel treatment strategies for early-stage lung cancer: The oncologist's perspective. J. Thorac. Dis. 2020, 12, 3390-3398. [CrossRef] [PubMed]

12. Majem, B.; Nadal, E.; Muñoz-Pinedo, C. Exploiting metabolic vulnerabilities of Non small cell lung carcinoma. Semin. Cell Dev. Biol. 2020, 98, 54-62. [CrossRef] [PubMed]

13. Rigas, J.R. Taxane-platinum combinations in advanced non-small cell lung cancer: A review. Oncologist 2004, 9 (Suppl. S2), 16-23. [CrossRef]

14. Frisone, D.; Friedlaender, A.; Malapelle, U.; Banna, G.; Addeo, A. A BRAF new world. Crit. Rev. Oncol. Hematol. 2020, 152, 103008. [CrossRef] [PubMed]

15. Gandalovičová, A.; Rosel, D.; Fernandes, M.; Veselý, P.; Heneberg, P.; Čermák, V.; Petruželka, L.; Kumar, S.; Sanz-Moreno, V.; Brábek, J. Migrastatics-Anti-metastatic and Anti-invasion Drugs: Promises and Challenges. Trends Cancer 2017, 3, 391-406. [CrossRef] [PubMed]

16. Čermák, V.; Dostál, V.; Jelínek, M.; Libusová, L.; Kovář, J.; Rösel, D.; Brábek, J. Microtubule-targeting agents and their impact on cancer treatment. Eur. J. Cell Biol. 2020, 99, 151075. [CrossRef] [PubMed]

17. Sciani, J.M.; Angeli, C.B.; Antoniazzi, M.M.; Jared, C.; Pimenta, D.C. Differences and similarities among parotoid macrogland secretions in South American toads: A preliminary biochemical delineation. Sci. World J. 2013, 2013, 937407. [CrossRef]

18. Schmeda-Hirschmann, G.; Quispe, C.; Arana, G.V.; Theoduloz, C.; Urra, F.A.; Cárdenas, C. Antiproliferative activity and chemical composition of the venom from the Amazonian toad Rhinella marina (Anura: Bufonidae). Toxicon 2016, 121, 119-129. [CrossRef]

19. Sousa, L.Q.; Machado, K.D.; Oliveira, S.F.; Araújo, L.D.; Monção-Filho, E.D.; Melo-Cavalcante, A.A.; Vieira-Júnior, G.M.; Ferreira, P.M. Bufadienolides from amphibians: A promising source of anticancer prototypes for radical innovation, apoptosis triggering and $\mathrm{Na}(+) / \mathrm{K}(+)$-ATPase inhibition. Toxicon 2017, 127, 63-76. [CrossRef]

20. Tornesello, A.L.; Borrelli, A.; Buonaguro, L.; Buonaguro, F.M.; Tornesello, M.L. Antimicrobial peptides as anticancer agents: Functional properties and biological activities. Molecules 2020, 25, 2850. [CrossRef]

21. Kunda, N.K. Antimicrobial peptides as novel therapeutics for non-small cell lung cancer. Drug Discov. Today 2020, 25, 238-247. [CrossRef]

22. Xia, F.; Gao, F.; Yao, H.; Zhang, G.; Gao, B.; Lu, Y.; Wang, X.; Qian, Y. Identification of angiogenesis-inhibiting peptides from Chan Su. Protein Expr. Purif. 2019, 163, 105445. [CrossRef] 
23. Han, Y.; Lu, M.; Zhou, J. Buforin Ilb induces androgen-independent prostate cancer cells apoptosis though p53 pathway in vitro. Toxicon 2019, 168, 16-21. [CrossRef] [PubMed]

24. Acevedo, A.A.; Lampo, M.; Cipriani, R. The cane or marine toad, Rhinella marina (Anura, Bufonidae): Two genetically and morphologically distinct species. Zootaxa 2016, 4103, 574-586. [CrossRef] [PubMed]

25. Orihuela-Torres, A.; Ordóñez-Delgado, L.; Brito, J.; López, F.; Mazón, M.; Freile, J.F. Feeding ecology of the Burrowing Owl Athene cunicularia punensis (Strigiformes: Strigidae) in the Jambelí archipelago, El Oro province, southwestern Ecuador. Rev. Peru. Biol. 2018, 25, 123-130. [CrossRef]

26. de Medeiros, D.S.S.; Rego, T.B.; Dos Santos, A.P.A.; Pontes, A.S.; Moreira-Dill, L.S.; Matos, N.B.; Zuliani, J.P.; Stábeli, R.G.; Teles, C.B.G.; Soares, A.M.; et al. Biochemical and Biological profile of parotoid secretion of the Amazonian Rhinella marina (Anura: Bufonidae). Biomed. Res. Int. 2019, 2019, 2492315. [CrossRef] [PubMed]

27. Kamano, Y.; Nogawa, T.; Yamashita, A.; Pettit, G. The $1 \mathrm{H}$ and $13 \mathrm{C}$ NMR chemical shift assignments for thirteen bufadienolides isolated from the traditional Chinese drug Ch'an Su. Collect. Czechoslov. Chem. Commun. 2001, 66, 1841-1848. [CrossRef]

28. Cao, Y.; Wu, J.; Pan, H.; Wang, L. Chemical profile and multicomponent quantitative analysis for the quality evaluation of toad venom from different origins. Molecules 2019, 24, 3595. [CrossRef] [PubMed]

29. Ye, M.; Guo, D.A. Analysis of bufadienolides in the Chinese drug ChanSu by high-performance liquid chromatography with atmospheric pressure chemical ionization tandem mass spectrometry. Rapid Commun. Mass Spectrom. 2005, 19, 1881-1892. [CrossRef] [PubMed]

30. Wei, W.L.; Hou, J.J.; Wang, X.; Yu, Y.; Li, H.J.; Li, Z.W.; Feng, Z.J.; Qu, H.; Wu, W.Y.; Guo, D.A. Venenum bufonis: An overview of its traditional use, natural product chemistry, pharmacology, pharmacokinetics and toxicology. J. Ethnopharmacol. 2019, 237, 215-235. [CrossRef] [PubMed]

31. Zhang, Y.; Jin, H.; Li, X.; Zhao, J.; Guo, X.; Wang, J.; Guo, Z.; Zhang, X.; Tao, Y.; Liu, Y.; et al. Separation and characterization of bufadienolides in toad skin using two-dimensional normal-phase liquid chromatography $\times$ reversed-phase liquid chromatography coupled with mass spectrometry. J. Chromatogr. B Anal. Technol. Biomed. Life Sci. 2016, 1026, 67-74. [CrossRef]

32. Liu, Y.; Xiao, Y.; Xue, X.; Zhang, X.; Liang, X. Systematic screening and characterization of novel bufadienolides from toad skin using ultra-performance liquid chromatography/electrospray ionization quadrupole time-of-flight mass spectrometry. Rapid Commun. Mass Spectrom. 2010, 24, 667-678. [CrossRef] [PubMed]

33. Ren, W.; Han, L.; Luo, M.; Bian, B.; Guan, M.; Yang, H.; Han, C.; Li, N.; Li, T.; Li, S.; et al. Multi-component identification and target cell-based screening of potential bioactive compounds in toad venom by UPLC coupled with high-resolution LTQ-Orbitrap MS and high-sensitivity Qtrap MS. Anal. Bioanal. Chem. 2018, 410, 4419-4435. [CrossRef] [PubMed]

34. Bessa-Silva, A.; Vallinoto, M.; Sampaio, I.; Flores-Villela, O.A.; Smith, E.N.; Sequeira, F. The roles of vicariance and dispersal in the differentiation of two species of the Rhinella marina species complex. Mol. Phylogenet. Evol. 2020, 145, 106723. [CrossRef] [PubMed]

35. Kamano, Y.; Yamashita, A.; Nogawa, T.; Morita, H.; Takeya, K.; Itokawa, H.; Segawa, T.; Yukita, A.; Saito, K.; Katsuyama, M.; et al. QSAR evaluation of the Ch'an Su and related bufadienolides against the colchicine-resistant primary liver carcinoma cell line PLC/PRF/5(1). J. Med. Chem. 2002, 45, 5440-5447. [CrossRef] [PubMed]

36. Nogawa, T.; Kamano, Y.; Yamashita, A.; Pettit, G.R. Isolation and structure of five new cancer cell growth inhibitory bufadienolides from the Chinese traditional drug Ch'an Su. J. Nat. Prod. 2001, 64, 1148-1152. [CrossRef]

37. Xu, R.; Xie, H.Q.; Deng, L.L.; Zhang, J.X.; Yang, F.M.; Liu, J.H.; Hao, X.J.; Zhang, Y.H. A new bufadienolide with cytotoxic activity from the Chinese traditional drug Ch'an Su. Chin. J. Nat. Med. 2014, 12, 623-627. [CrossRef]

38. Kim, N.Y.; Suh, Y.A.; Kim, S.; Lee, C. Bufalin down-regulates Axl expression to inhibit cell proliferation and induce apoptosis in non-small-cell lung cancer cells. Biosci. Rep. 2020, 40. [CrossRef] 
39. Wang, J.; Cai, H.; Liu, Q.; Xia, Y.; Xing, L.; Zuo, Q.; Zhang, Y.; Chen, C.; Xu, K.; Yin, P.; et al. Cinobufacini Inhibits Colon Cancer Invasion and Metastasis via Suppressing Wnt/ $\beta$-Catenin Signaling Pathway and EMT. Am. J. Chin. Med. 2020, 48, 703-718. [CrossRef]

40. Zhao, J.; Zhang, Q.; Zou, G.; Gao, G.; Yue, Q. Arenobufagin, isolated from toad venom, inhibited epithelial-to-mesenchymal transition and suppressed migration and invasion of lung cancer cells via targeting IKK $\beta / N F K B$ signal cascade. J. Ethnopharmacol. 2020, 250, 112492. [CrossRef]

41. Franken, N.A.; Rodermond, H.M.; Stap, J.; Haveman, J.; van Bree, C. Clonogenic assay of cells in vitro. Nat. Protoc. 2006, 1, 2315-2319. [CrossRef]

42. Jeyapalan, J.; Leake, A.; Ahmed, S.; Saretzki, G.; Tilby, M.; von Zglinicki, T. The role of telomeres in Etoposide induced tumor cell death. Cell Cycle 2004, 3, 1169-1176. [CrossRef]

43. Jain, C.K.; Roychoudhury, S.; Majumder, H.K. Selective killing of G2 decatenation checkpoint defective colon cancer cells by catalytic topoisomerase II inhibitor. Biochim. Biophys. Acta 2015, 1853, 1195-1204. [CrossRef] [PubMed]

44. Clifford, B.; Beljin, M.; Stark, G.R.; Taylor, W.R. G2 arrest in response to topoisomerase II inhibitors: The role of p53. Cancer Res. 2003, 63, 4074-4081.

45. Chiu, C.C.; Li, C.H.; Ung, M.W.; Fuh, T.S.; Chen, W.L.; Fang, K. Etoposide (VP-16) elicits apoptosis following prolonged G2-M cell arrest in p53-mutated human non-small cell lung cancer cells. Cancer Lett. 2005, 223, 249-258. [CrossRef]

46. Ceballos-Cancino, G.; Espinosa, M.; Maldonado, V.; Melendez-Zajgla, J. Regulation of mitochondrial Smac/DIABLO-selective release by survivin. Oncogene 2007, 26, 7569-7575. [CrossRef] [PubMed]

47. Meresse, P.; Dechaux, E.; Monneret, C.; Bertounesque, E. Etoposide: Discovery and medicinal chemistry. Curr. Med. Chem. 2004, 11, 2443-2466. [CrossRef] [PubMed]

48. Ma, L.; Song, B.; Jin, H.; Pi, J.; Liu, L.; Jiang, J.; Cai, J. Cinobufacini induced MDA-MB-231 cell apoptosis-associated cell cycle arrest and cytoskeleton function. Bioorg. Med. Chem. Lett. 2012, 22, 1459-1463. [CrossRef]

49. Hashimoto, S.; Jing, Y.; Kawazoe, N.; Masuda, Y.; Nakajo, S.; Yoshida, T.; Kuroiwa, Y.; Nakaya, K. Bufalin reduces the level of topoisomerase II in human leukemia cells and affects the cytotoxicity of anticancer drugs. Leuk. Res. 1997, 21, 875-883. [CrossRef]

50. Liu, Y.; Ban, L.Y.; Su, X.; Gao, S.; Liu, J.W.; Cui, X.N. Effects of cinobufacini injection on cell proliferation and the expression of topoisomerases in human HepG-2 hepatocellular carcinoma cells. Mol. Med. Rep. 2015, 12, 1598-1604. [CrossRef] [PubMed]

51. Deng, L.J.; Peng, Q.L.; Wang, L.H.; Xu, J.; Liu, J.S.; Li, Y.J.; Zhuo, Z.J.; Bai, L.L.; Hu, L.P.; Chen, W.M.; et al. Arenobufagin intercalates with DNA leading to G2 cell cycle arrest via ATM/ATR pathway. Oncotarget 2015, 6, 34258-34275. [CrossRef]

52. Zhang, Y.; Yuan, B.; Takagi, N.; Wang, H.; Zhou, Y.; Si, N.; Yang, J.; Wei, X.; Zhao, H.; Bian, B. Comparative analysis of hydrophilic ingredients in toad skin and toad venom using the UHPLC-HR-MS/MS and UPLC-QqQ-MS/MS methods together with the anti-inflammatory evaluation of indolealkylamines. Molecules 2018, 24, 86. [CrossRef]

53. Soumoy, L.; Wells, M.; Najem, A.; Krayem, M.; Ghanem, G.; Hambye, S.; Saussez, S.; Blankert, B.; Journe, F. Toad venom antiproliferative activities on metastatic melanoma: Bio-guided fractionation and screening of the compounds of two different venoms. Biology 2020, 9, 218. [CrossRef] [PubMed]

54. Sinhorin, A.P.; Kerkhoff, J.; Dall'Oglio, E.L.; de Jesus Rodrigues, D.; de Vasconcelos, L.G.; Sinhorin, V.D.G. Chemical profile of the parotoid gland secretion of the Amazonian toad (Rhinella margaritifera). Toxicon 2020, 182, 30-33. [CrossRef] [PubMed]

55. Zhan, X.; Wu, H.; Wu, H.; Wang, R.; Luo, C.; Gao, B.; Chen, Z.; Li, Q. Metabolites from Bufo gargarizans (Cantor, 1842): A review of traditional uses, pharmacological activity, toxicity and quality control. J. Ethnopharmacol. 2020, 246, 112178. [CrossRef] [PubMed]

56. Thirupathi, K.; Chandrakala, G.; Krishna, L.; Bheem Rao, T.; Venkaiah, Y. Antibacterial activity of skin secretion and its extraction from the toad Bufo melanostictus. Eur. J. Pharm. Med Res. 2018, 3, 283-286. 
57. Fuentes-Retamal, S.; Sandoval-Acuna, C.; Peredo-Silva, L.; Guzman-Rivera, D.; Pavani, M.; Torrealba, N.; Truksa, J.; Castro-Castillo, V.; Catalan, M.; Kemmerling, U.; et al. Complex Mitochondrial Dysfunction Induced by TPP(+)-Gentisic Acid and Mitochondrial Translation Inhibition by Doxycycline Evokes Synergistic Lethality in Breast Cancer Cells. Cells 2020, 9, 407. [CrossRef]

58. Urra, F.A.; Munoz, F.; Cordova-Delgado, M.; Ramirez, M.P.; Pena-Ahumada, B.; Rios, M.; Cruz, P.; Ahumada-Castro, U.; Bustos, G.; Silva-Pavez, E.; et al. FR58P1a; a new uncoupler of OXPHOS that inhibits migration in triple-negative breast cancer cells via Sirt1/AMPK/beta1-integrin pathway. Sci. Rep. 2018, 8, 13190. [CrossRef]

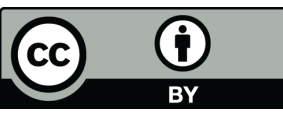

(C) 2020 by the authors. Licensee MDPI, Basel, Switzerland. This article is an open access article distributed under the terms and conditions of the Creative Commons Attribution (CC BY) license (http://creativecommons.org/licenses/by/4.0/). 